\title{
Verifying Message-Passing Programs with Dependent Behavioural Types
}

\author{
Alceste Scalas \\ Imperial College London \\ and Aston University, Birmingham \\ UK \\ a.scalas@aston.ac.uk
}

\author{
Nobuko Yoshida \\ Imperial College London \\ UK \\ n.yoshida@imperial.ac.uk
}

\author{
Elias Benussi \\ Imperial College London \\ and Faculty Science Ltd. \\ UK \\ elias@faculty.ai
}

\begin{abstract}
Concurrent and distributed programming is notoriously hard. Modern languages and toolkits ease this difficulty by offering message-passing abstractions, such as actors (e.g., Erlang, Akka, Orleans) or processes (e.g., Go): they allow for simpler reasoning w.r.t. shared-memory concurrency, but do not ensure that a program implements a given specification.

To address this challenge, it would be desirable to specify and verify the intended behaviour of message-passing applications using types, and ensure that, if a program type-checks and compiles, then it will run and communicate as desired.

We develop this idea in theory and practice. We formalise a concurrent functional language $\lambda_{\leqslant}^{\pi}$, with a new blend of behavioural types (from $\pi$-calculus theory), and dependent function types (from the Dotty programming language, a.k.a. the future Scala 3). Our theory yields four main payoffs: (1) it verifies safety and liveness properties of programs via typelevel model checking; (2) unlike previous work, it accurately verifies channel-passing (covering a typical pattern of actor programs) and higher-order interaction (i.e., sending/receiving mobile code); (3) it is directly embedded in Dotty, as a toolkit called Effpi, offering a simplified actor-based API; (4) it enables an efficient runtime system for Effpi, for highly concurrent programs with millions of processes/actors.
\end{abstract}

CCS Concepts - Theory of computation $\rightarrow$ Process calculi; Type structures; Verification by model checking; • Software and its engineering $\rightarrow$ Concurrent programming languages.

Keywords behavioural types, dependent types, processes, actors, Dotty, Scala, temporal logic, model checking

\section{ACM Reference Format:}

Alceste Scalas, Nobuko Yoshida, and Elias Benussi. 2019. Verifying Message-Passing Programs with Dependent Behavioural Types . In

PLDI '19, fune 22-26, 2019, Phoenix, AZ, USA

(C) 2019 Copyright held by the owner/author(s). Publication rights licensed to ACM.

This is the author's version of the work. It is posted here for your personal use. Not for redistribution. The definitive Version of Record was published in Proceedings of the 40th ACM SIGPLAN Conference on Programming Language Design and Implementation (PLDI '19), June 22-26, 2019, Phoenix, AZ, USA, https://doi.org/10.1145/3314221.3322484.
Proceedings of the 40th ACM SIGPLAN Conference on Programming Language Design and Implementation (PLDI '19), June 22-26, 2019, Phoenix, AZ, USA. ACM, New York, NY, USA, 15 pages. https://doi. org/10.1145/3314221.3322484

\section{Introduction}

Consider this specification for a payment service with auditing (from a use case for the Akka Typed toolkit [42, 50]):

1. the service waits for Pay messages, carrying an amount; 2. the service can decide to either:

a. reject the payment, by sending Rejected to the payer;

b. accept the payment. Then, it must report it to an auditing service, and send Accepted to the payer;

3. then, the service loops to 1 , to handle new Payments.

This can be implemented using various languages and tools for concurrent and distributed programming. E.g., using Scala and Akka Typed [50], a developer can write a solution similar to Fig. 1: payment is an actor, receiving messages of type Pay (line 1); aud is the actor reference of the auditor, used to send messages of type Audit; whenever a pay message is received (line 3), payment checks the amount (line 4), and uses the pay.replyTo field to answer either Accepted or Rejected - notifying the auditor in the first case.

The typed actor references in Fig. 1 guarantee type safety: e.g., writing send(aud, "Hi") causes a compilation error. However, the payment service specification is not enforced: e.g., if the developer forgets to write line 7, the code still compiles, but accepted payments are not audited. This is a typical concurrency bug: a missing or out-of-order communication can cause protocol violations, deadlocks, or livelocks. Such bugs are often spotted late, during software testing or maintenance - when they are more difficult to find and fix, and harmful: e.g., what if unaudited payments violate fiscal rules?

These issues were considered during the design of Akka Typed, with the idea of using types for specifying protocols [46], and produce compilation errors when a program violates a desired protocol. However, the resulting experiments [41] had no rigorous grounding: although inspired by the session types theory $[3,26]$, the approach was informal, and the kind of assurances that it could provide are unclear. Still, the idea has intriguing potential: if realised, it would allow to check the payment specification above at compile-time. 


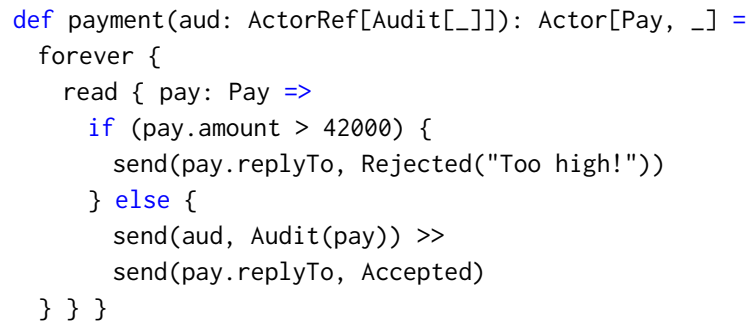

Figure 1. Implemention of the payment service specification (§1). Although similar to Akka Typed [50], it is written in Dotty and Effpi, described in $§ 5$; “>” (1.7) means "and then."

Our proposal is a new take on specifying and statically verifying the behaviour of concurrent programs, in two steps.

Step 1: enforcing protocols at compile-time We develop Effpi [64], a toolkit for message-passing programming in Dotty (a.k.a. Scala 3), that allows to verify the code in Fig. 1 against its specification, at compile time. This is achieved by replacing the rightmost "_" (line 1) with a behavioural type:

Forever[ In[Pay, (p: Pay) $\Rightarrow>/ /$ Dependent function type [16] Out[p.replyTo.type, Rejected]

| ( Out[aud.type, Audit[p.type]] >>: Out [p.replyTo.type, Accepted] ) ] ]

With this type annotation, the code in Fig. 1 still type-checks and compiles; but if, e.g., line 7 is forgotten, or changed in a way that does not audit properly (e.g., writing null instead of aud), then a compilation error ensues. The type above formalises the payment service specification by capturing the desired behaviour of its implementation, and tracking which ActorReferences are used for interacting, and when. Type "In" (provided by Effpi) requires to wait for a message $p$ of type Pay, and then either (| means "or") send Rejected on p. replyTo, or send an audit, and then (>>:) send Accepted. Notably, $p$ is bound by a dependent function type [16]

Effpi is built upon a concurrent functional calculus for channel-based interaction, called $\lambda_{\leqslant}^{\pi}$; its novelty is a blend of behavioural types (inspired by $\pi$-calculus literature) with dependent function types (inspired by Dotty's foundation $D_{<\text {: }}$ [2]), achieving unique specification and verification capabilities. Effpi implements $\lambda_{\leqslant}^{\pi}$ as an internal DSL in Dotty plus syntactic sugar for an actor-based API (cf. Fig. 1).

Step 2: verification of safety/liveness properties In Step 1 , we establish the correspondence between protocols and programs, via syntax-driven typing rules. But this is not enough: programs may be expected to have safety properties ("unwanted events never happen") or liveness properties ("desired events will happen") [43]. E.g., in our example, we want each accepted payment to be audited; but in principle, an auditor's implementation might be based on a type like:

In[ Audit[_], (a: Audit[_]) $\Rightarrow$ End ]

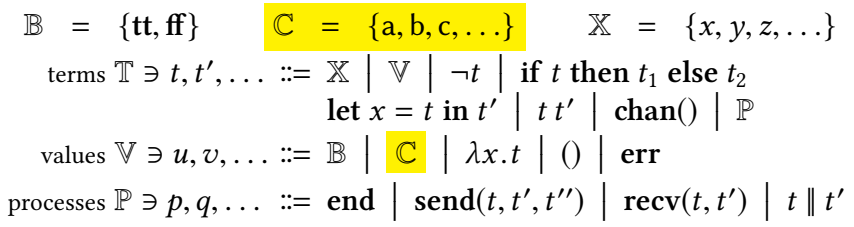

Figure 2. Syntax of $\lambda_{\leqslant}^{\pi}$ terms. The set $\mathbb{C}$ (highlighted) contains channel instances, that are part of the run-time syntax.

(i.e., receive one Audit message a, and terminate). This implementation, in isolation, may be deemed correct by mere type checking; however, if such an auditor is composed with the payment service above (receiving messages sent on aud), the resulting application would not satisfy the desired property: only one accepted payment is audited. With complex protocols, similar problems become more difficult to spot.

The issue is that types in $\lambda_{\leq}^{\pi}$ and $\mathrm{Effpi}$ can specify rich protocols - but when such protocols (and their implementations) are composed, they might yield undesired behaviours. Hence, we develop a method to: (1) compose types/protocols, and decide whether they enjoy safety / liveness properties; (2) transfer behavioural properties of types to programs.

Contribution We present a new method to develop message-passing programs with verified safety/liveness properties, via type-level model checking. The key insight is: we use variables in types, to track inputs/outputs in programs, through a novel blend of behavioural+dependent function types. Unlike previous work, our theory can track channels across transmissions, and verify mobile code, covering important features of modern message-passing programs.

Outline. $\$ 2$ formalises the $\lambda_{\leqslant}^{\pi}$ calculus, at the basis of Effpi. $\$ 3$ presents type system of $\lambda_{\leqslant}^{\pi}$. $\$ 4$ shows the correspondence between type / process transitions (Thm. 4.4, 4.5), and how to transfer temporal logic judgements on types (that are decidable, by Lemma 4.7) to processes. This yields Thm. 4.10: our new method to verify safety / liveness properties of programs. $\$ 5$ explains how the design of $\lambda_{\leqslant}^{\pi}$ naturally leads Effpi's implementation (i.e., the paper's companion artifact), and evaluates: (1) its run-time performance and memory use (compared with Akka Typed); (2) the speed of type-level model checking. \$6 discusses related work. The technical report [70] contains proofs and more material.

\section{The $\lambda_{\leqslant}^{\pi}$-Calculus}

The theoretical basis of our work is a $\lambda$-calculus extended with channels, input/output, and parallel composition, called $\lambda \pi$. The " $\pi$ " denotes both: (1) its use of dependent function types, that, together with subtyping $\leqslant$, are cornerstones of its typing system ( $\$ 3$ ); and (2) its connection with the $\pi$-calculus $[54,55,63]$. Indeed, $\lambda_{\leqslant}^{\pi}$ is a monadic-style encoding of the higher-order $\pi$-calculus: continuations are $\lambda$-terms, and this will be helpful for typing (§3) and implementation (§5). 
Definition 2.1. The syntax of $\lambda_{\leqslant}^{\pi}$ is in Fig. 2. Elements of $\mathbb{C}$ are run-time syntax. Free/bound variables $\mathrm{fv}(t) / \mathrm{bv}(t)$ are defined as usual. We adopt the Barendregt convention: bound variables are syntactically distinct from each other, and from free variables. We write $\lambda_{-} . t$ for $\lambda x . t$, when $x \notin \mathrm{fv}(t)$.

The set of values $\mathbb{V}$ includes booleans $\mathbb{B}$, channel instances $\mathbb{C}$, function abstraction, the unit (), and error. The terms (in $\mathbb{T}$ ) can be variables (from $\mathbb{X}$ ), values (from $\mathbb{V}$ ), various standard constructs (negation $\neg t$, if/then/else, let binding, function application), and also channel creation chan(), and process terms (from $\mathbb{P}$ ). The primitive chan $($ ) evaluates by returning a fresh channel instance from $\mathbb{C}$ - whose elements are part of the run-time syntax, and cannot be written by programmers. Process terms include the terminated process end, the output primitive send $\left(t, t^{\prime}, t^{\prime \prime}\right)$ (meaning: send $t^{\prime}$ through $t$, and continue as $\left.t^{\prime \prime}\right)$, the input primitive $\operatorname{recv}\left(t, t^{\prime}\right)$ (meaning: receive a value from $t$, and continue as $t^{\prime}$ ), and the parallel composition $t \| t^{\prime}$ (meaning: $t$ and $t^{\prime}$ run concurrently, and can interact). $\lambda_{\leqslant}^{\pi}$ can be routinely extended with, e.g., integers, strings, records, variants: we use them in examples.

Example 2.2. A ping-pong system in $\lambda_{\leqslant}^{\pi}$ is written as:

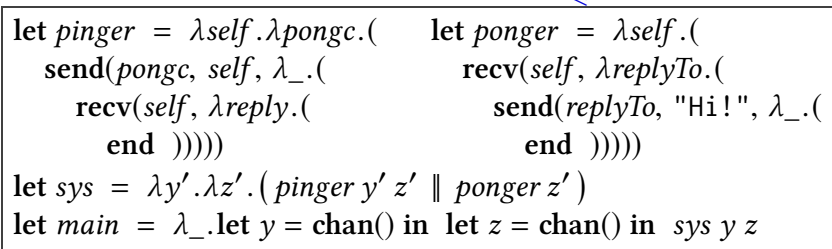

- pinger is an abstract process that takes two channels: self (its own input channel), and pongc. It uses pongc to send self, then uses self to receive a response, and ends;

- ponger takes a channel self, uses it to receive replyTo, then uses replyTo to send "Hi !", and ends;

- sys takes channels $y^{\prime}, z^{\prime}$, and uses them to instantiate pinger and ponger in parallel;

- invoking main() instantiates sys with $y$ and $z$ (containing channel instances): this lets pinger and ponger interact.

Note that in pinger and ponger, the last argument of send/recv is always an abstract process term: this is expected by the semantics (Def. 2.4), and enforced via typing (§3).

Remark 2.3. In Ex. 2.2, pinger / ponger use channel passing to realise a typical pattern of actor programs: they have their own "mailbox" (self), and interact by exchanging their own "reference" (again, self). We will leverage this intuition in $\$ 5$.

Definition 2.4 (Semantics of $\lambda_{\leqslant}^{\pi}$ ). Evaluation contexts $\mathcal{E}$ and reduction $\rightarrow$ are illustrated in Fig. 3, where congruence 三 is defined as: $t_{1}\left\|t_{2} \equiv t_{2}\right\| t_{1}$ and end $\|$ end $\equiv$ end, plus $\alpha$-conversion. We write $\rightarrow^{*}$ for the reflexive and transitive closure of $\rightarrow$. We say " $t$ has an error" iff $t=\mathcal{E}$ [err] (for some $\mathcal{E})$. We say " $t$ is safe" iff $\forall t^{\prime}: t \rightarrow^{*} t^{\prime}$ implies $t^{\prime}$ has no error.

Def. 2.4 is a standard call-by-value semantics, with two rules for concurrency. [R-chan()] says that $\operatorname{chan}()$ returns a fresh channel instance; [R-Сомм] says that the parallel composition $\operatorname{send}\left(\mathrm{a}, u, v_{1}\right) \| \operatorname{recv}\left(\mathrm{a}, v_{2}\right)$, where both sides operate on a same channel instance a, transfers the value $u$ on the receiver side, yielding $v_{1}() \| v_{2} u$ : hence, if $v_{1}$ and $v_{2}$ are function values, the process keeps running by applying $v_{1}()$ and $v_{2} u$-i.e. the sent value is substituted inside $v_{2}$. The error rules say how terms can "go wrong:" they include usual type mismatches (e.g., it is an error to apply a non-function value $u$ to any $v$ ), and three rules for concurrency: it is an error to receive/send data using a value $u$ that is not a channel, and it is an error to put a value in a parallel composition (i.e., only processes from $\mathbb{P}$ in Fig. 2 are safely composed by $\|$ ).

\section{Type System}

We now introduce the type system of $\lambda_{\leqslant}^{\pi}$. Its design is reminiscent of the simply-typed $\lambda$-calculus, except that (1) we include union types and equi-recursive types, (2) we add types for channels and processes, and (3) we allow types to contain variables from the term syntax (inspired by $D_{<:}$, the calculus behind Dotty [2]). The syntax of types is in Def. 3.1.

Notably, points (1) and (3) establish a similarity between $\lambda \leqslant$ and $F_{<:}$(System $F$ with subtyping [8]) equipped with equi-recursive types [32]. Indeed, point (3) means that a type $T$ is only valid if its variables exist in the typing environment - which, in turn, must contain valid types. Similarly, in $F_{<:}$, polymorphic types can depend on type variables in the environment; hence, we use mutually-defined judgements, akin to those of $F_{<:}$, to assess the validity of environments, types, subtyping, and typed terms (Def. 3.2).

Definition 3.1 (Syntax of types). Types, ranged over by $S, T, U, \ldots$, are inductively defined by the productions:

$$
\begin{aligned}
& \text { bool }|()| \mathrm{T}|\perp| T \vee U|\Pi(\underline{x}: U) T| \mu \underline{x} . T \mid \underline{x} \\
&{ }_{\mathrm{c}^{\mathrm{i}}}[T]\left|\mathrm{c}^{\mathrm{i}}[T]\right| \mathrm{c}^{\mathrm{O}}[T] \\
& \text { proc } \mid \text { nil }|\mathbf{o}[S, T, U]| \mathrm{i}[S, T] \mid \mathrm{p}[T, U]
\end{aligned}
$$

Free/bound variables are defined as usual. We write $U\{S / \underline{x}\}$ for the type obtained from $U$ by replacing its free occurrences of $\underline{x}$ with $S$. If $T=\Pi\left(\underline{x}: U^{\prime}\right) U$, then $T S$ stands for $U\{S / \underline{x}\}$.

We write $\Pi() T$ for $\Pi(\underline{x}:()) T$ if $\underline{x} \notin \mathrm{fv}(T)$, and distinguish recursion variables as $\mathbf{t}, \mathbf{t}^{\prime}, \ldots$ (i.e., we write $\mu \mathbf{t} . T$ ). We write $\widetilde{T}$ for an $n$-tuple $T_{1}, \ldots, T_{n}$, and $T \in U$ if $T$ occurs in $U$.

The relation $\equiv$ is the smallest congruence such that:

$T \vee U \equiv U \vee T \quad S \vee(T \vee U) \equiv(S \vee T) \vee U \quad \mu \mathbf{t} . T \equiv T\{\mu \mathbf{t} T / \mathbf{t}\}$ $\mathbf{p}[T, U] \equiv \mathbf{p}[U, T] \quad \mathbf{p}[S, \mathbf{p}[T, U]] \equiv \mathbf{p}[\mathbf{p}[S, T], U] \quad \mathbf{p}[T, \mathbf{n i l}] \equiv T$

The first row of productions in Def. 3.1 includes booleans, the unit type (), top/bottom types $\top / \perp$, the union type $T \vee U$, the dependent function type $\Pi(\underline{x}: U) T$ and the recursive type $\mu \underline{x} . T$ (they both bind $\underline{x}$ with scope $T$ ), and variables $\underline{x}$ (from the set $\mathbb{X}$ in Def. 2.1): the underlining is a visual clue to better distinguish $\underline{x}$ used in a type, from $x$ used in a $\lambda_{\leqslant}^{\pi}$ term.

The second row of Def. 3.1 formalises channel types: $\mathrm{c}^{\text {io }}[T]$ denotes a channel allowing to input or output values of type $T$; instead, $\mathrm{c}^{\mathrm{i}}[T]$ only allows for input, and $\mathrm{c}^{\mathrm{o}}[T]$ for output. 


$$
\begin{aligned}
& \mathcal{E}::=[]|\neg \mathcal{E}| \text { if } \mathcal{E} \text { then } t_{1} \text { else } t_{2} \mid \text { let } x=\mathcal{E} \text { in } t \mid \text { let } x=w \text { in } \mathcal{E}|\mathcal{E} t| w \mathcal{E} \\
& \operatorname{send}\left(\mathcal{E}, t, t^{\prime}\right)\left|\operatorname{send}\left(w, \mathcal{E}, t^{\prime}\right)\right| \operatorname{send}\left(w, w^{\prime}, \mathcal{E}\right)|\operatorname{recv}(\mathcal{E}, t)| \operatorname{recv}(w, \mathcal{E}) \mid \mathcal{E} \| t \quad\left(\text { where } w, w^{\prime} \in \mathbb{V} \cup \mathbb{X}\right)
\end{aligned}
$$

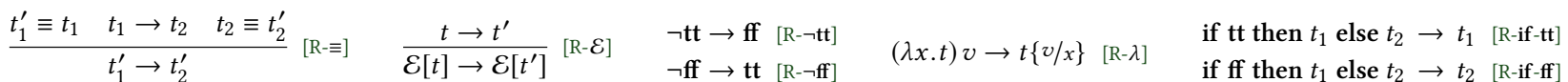

$$
\begin{aligned}
& \frac{w \in \mathbb{V} \cup \mathbb{X}}{\mathcal{E}[x] \rightarrow \text { let } x=w \text { in } \mathcal{E}[w]}[\mathrm{R}-\mathrm{let}] \quad \frac{x \notin \mathrm{fv}(t)}{\operatorname{let} x=w \text { in } t \rightarrow t}[\text { R-letGc }] \quad \frac{\mathrm{a} \text { fresh }}{\operatorname{chan}() \rightarrow \mathrm{a}}[\mathrm{R}-\operatorname{chan}()] \quad \frac{\mathrm{R}-\mathrm{Comm}]}{\operatorname{send}\left(\mathrm{a}, u, v_{1}\right)\left\|\operatorname{recv}\left(\mathrm{a}, v_{2}\right) \rightarrow v_{1}()\right\| v_{2} u} \\
& \frac{v \notin \mathbb{B}}{\neg v \rightarrow \operatorname{err}} \quad \frac{u \notin\{\lambda x . t \mid x \in \mathbb{X}, t \in \mathbb{T}\}}{u v \rightarrow \text { err }} \quad \frac{v \notin \mathbb{B}}{\text { if } v \text { then } t^{\prime} \text { else } t^{\prime \prime} \rightarrow \text { err }} \quad \frac{u \notin \mathbb{C}}{\operatorname{recv}(u, v) \rightarrow \text { err }} \quad \frac{u \notin \mathbb{C}}{\operatorname{send}\left(u, v_{1}, v_{2}\right) \rightarrow \operatorname{err}} \quad \frac{t \in \mathbb{V}}{t \| t^{\prime} \rightarrow \operatorname{err}}
\end{aligned}
$$

Figure 3. Semantics of $\lambda_{\leqslant}^{\pi}$ : evaluation contexts $\mathcal{E}$ (top), reduction rules (middle), and error rules (last row).

The third row of Def. 3.1 formalises process types. The generic process type proc denotes any process term; nil denotes a terminated process; the output type o[S,T,U] denotes a process that sends a $T$-typed value on an $S$-typed channel, and continues as $U$; the input type $\mathrm{i}[S, T]$ denotes a process that receives a value from an $S$-typed channel and continues as $T$; the parallel type $\mathrm{p}[T, U]$ denotes the parallel composition of two processes (of types $T$ and $U$ ).

Definition 3.2. These judgements are formalised in Fig. 4:

$\vdash \Gamma$ env

$\Gamma \vdash T$ type

$\Gamma$ is a valid typing environment

$\Gamma \vdash \widetilde{T}$ type

$T$ is a valid type in $\Gamma$

$\Gamma \vdash T \pi$-type

holds iff $\forall U \in \widetilde{T}: \Gamma \vdash U$ type

$\Gamma \vdash \widetilde{T} \pi$-type

$T$ is a valid process type in $\Gamma$

$\Gamma \vdash \widetilde{T}^{*}$-type

holds iff $\forall U \in \widetilde{T}: \Gamma \vdash U \pi$-type

$\Gamma \vdash T \leqslant U$

holds if $\Gamma \vdash \widetilde{T}$ type or $\Gamma \vdash \widetilde{T} \pi$-type

$\Gamma \vdash t: T$

$T$ is subtype of $U$ in $\Gamma$, if $\Gamma \vdash T, U$ *type

$t$ has type $T$ in $\Gamma$

A typing environment $\Gamma$ maps variables (from $\mathbb{X}$ in Def. 2.1) to types; the order of the entries of $\Gamma$ is immaterial. All judgements in Fig. 4 are inductive, except subtyping, that is coinductive (hence the double inference lines). Crucially, in Fig. 4 we have $t w o$ valid type judgements, for two kinds of types: $\Gamma \vdash T$ type and $\Gamma \vdash T \pi$-type. The former is standard (except for rule $[T-\mathrm{c}]$, for valid channel types); the latter distinguishes process types. Note that subtyping only relates types of the same kind. Importantly, a typing environment $\Gamma$ can map a variable to a type (rule $[\Gamma-x]$ ), but not to a $\pi$-type; this also means that function arguments cannot be $\pi$-typed. Still, in a function type $\Pi(\underline{x}: T) U$, the return type $U$ can be a $\pi$ type (rule $[T \pi-\Pi])$ : i.e., it is possible to define abstract process types (cf. Ex. 3.3 and 3.4 later). Rules $[T-\mu]$ and $[\pi-\mu]$ are based on $[32, \S 2]$, and require recursive types to be contractive: e.g., $\mu \mathbf{t}_{1} . \mu \mathbf{t}_{2} \ldots \mu \mathbf{t}_{n} .\left(\mathbf{t}_{1} \vee U\right)$ is not a type; clause " $\underline{x} \notin \mathrm{fv}^{-}(T)$ " means that variable $x$ is not bound in negative position in $T$, as in $F_{<:}$(Details: [70]). Recursion is handled by [ $t$-let]: in

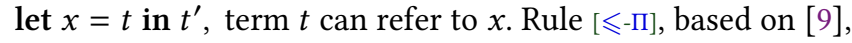
ensures decidability of subtyping $[32, \S 1]$ : it is often needed in practice, and we use it in Def. 4.2, Lemma 4.7. The rest of Fig. 4 is standard; we discuss the main judgements.

Variables, types, subtyping, and dependencies The environment $\Gamma=x: T$ assigns type $T$ to variable $x$. Hence, by rule $[T-\underline{x}]$, the type $\underline{x}$ is valid in $\Gamma$; and indeed, by rule $[t-x]$, we can infer $\Gamma \vdash x: x$, i.e., the term $x$ has type $x$. Intuitively, this means that $x$ is the "most precise" type for term $x$; this is formally supported by the subtyping rule $[t-x]$, that says: as $\Gamma$ maps term $x$ to $T$, type $x$ is smaller than $T$. To retrieve from $\Gamma$ the information that term $x$ has (also) type $T$, we use subtyping and subsumption (rule $[t-\leqslant]$ ), as shown here. Since

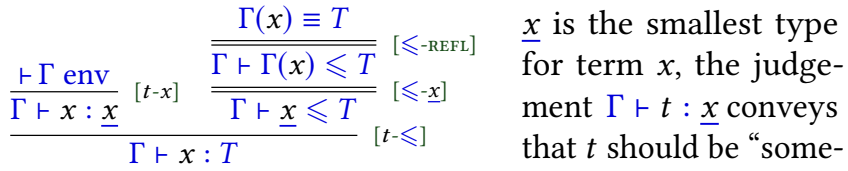
thing that evaluates to $x$, e.g., $t=x$ or $t=$ if tt then $x$ else $x$; similarly, the dependent function type $\Pi(\underline{x}$ :bool $) \underline{x}$ is inhabited by terms like $\lambda x \cdot x$ or $\lambda x .(\lambda y \cdot y) x$. Thus, we can roughly say: if $x$ occurs in $T$, then $T$-typed terms correspondingly use $x$. This insight will be crucial for our results.

Channels, processes, and their types By [t-chan], a (typeannotated) term chan ()$^{T}$ has type $c^{\text {io }}[T]$. Rule $[t-\mathbb{C}]$ is similar, for channel instances. By [t-end], process end has type nil.

By [t-\|], both sub-terms of $t_{1} \| t_{2}$ are $\pi$-typed.

By $\left[t\right.$-send], send $\left(t_{1}, t_{2}, t_{3}\right)$ has type o[ $\left.S, T, U\right]$, under the validity constraints of rule $[\pi-0]$. Hence, $t_{1}$ has a channel type for sending values of type $T$, and $t_{2}$ (the term being sent) must have type $T$; also, $t_{3}$ 's type must be $U=\Pi() U^{\prime}$ (for a $\pi$-type $U^{\prime}$ ): i.e., $t_{3}$ is a process thunk, run by applying $t_{3}()$.

By $\left[t\right.$-recv], $\operatorname{recv}\left(t_{1}, t_{2}\right)$ has type $\mathrm{i}[S, T]$, which is well-formed under rule $[\pi-\mathrm{i}]$. Hence, the sub-term $t_{1}$ must have a channel type with input $U$, while $t_{2}$ must be an abstract process of type $T=\Pi\left(\underline{x}: U^{\prime}\right) T^{\prime}$, with $T^{\prime} \pi$-type. Crucially, by rule $[\pi-\mathrm{i}]$, we have $\Gamma \vdash \bar{U} \leqslant U^{\prime}$ : hence, it is safe to receive a value $v$ from $t_{1}$, and apply $t_{2} v$ to get a continuation process that uses $v$.

We explain subtyping in Fig. 4 later, after a few examples.

Example 3.3. In Ex. 2.2, we have the type assignments:

$$
\begin{aligned}
& \text { pinger }: T_{\text {ping }}=\Pi\left(\text { self }: \mathrm{c}^{\mathrm{io}}[\mathrm{str}]\right) \Pi\left(\text { pongc }: \mathrm{c}^{\mathrm{O}}\left[\mathrm{c}^{\mathrm{O}}[\mathrm{str}]\right]\right) \\
& \text { o[pongc, self }, \mathrm{i}[\text { self }, \Pi(\text { reply:str)nil }]] \\
& \text { ponger }: T_{\text {pong }}=\Pi\left(\text { self }: \mathrm{c}^{\mathrm{io}}\left[\mathrm{c}^{\mathrm{O}}[\mathrm{str}]\right]\right) \\
& \mathbf{i}\left[\underline{s e l f}, \Pi\left(\underline{\text { replyTo: }} \mathrm{c}^{\mathrm{o}}[\mathrm{str}]\right) \text { o }[\underline{\text { replyTo}}, \mathrm{str}, \Pi() \mathrm{nil}]\right] \\
& \text { sys }: T_{p p}=\Pi\left(\underline{y}: \mathrm{c}^{\mathrm{io}}[\operatorname{str}]\right) \Pi\left(\underline{z}: \mathrm{c}^{\mathrm{io}}\left[\mathrm{c}^{\mathrm{o}}[\mathrm{str}]\right]\right) \mathrm{p}\left[T_{\text {ping }} \underline{y} \underline{z}, T_{\text {pong }} \underline{z}\right]
\end{aligned}
$$

Notice how $T_{p p}$ captures the ping/pong composition of sys, preserving its channel topology: the type-level applications 


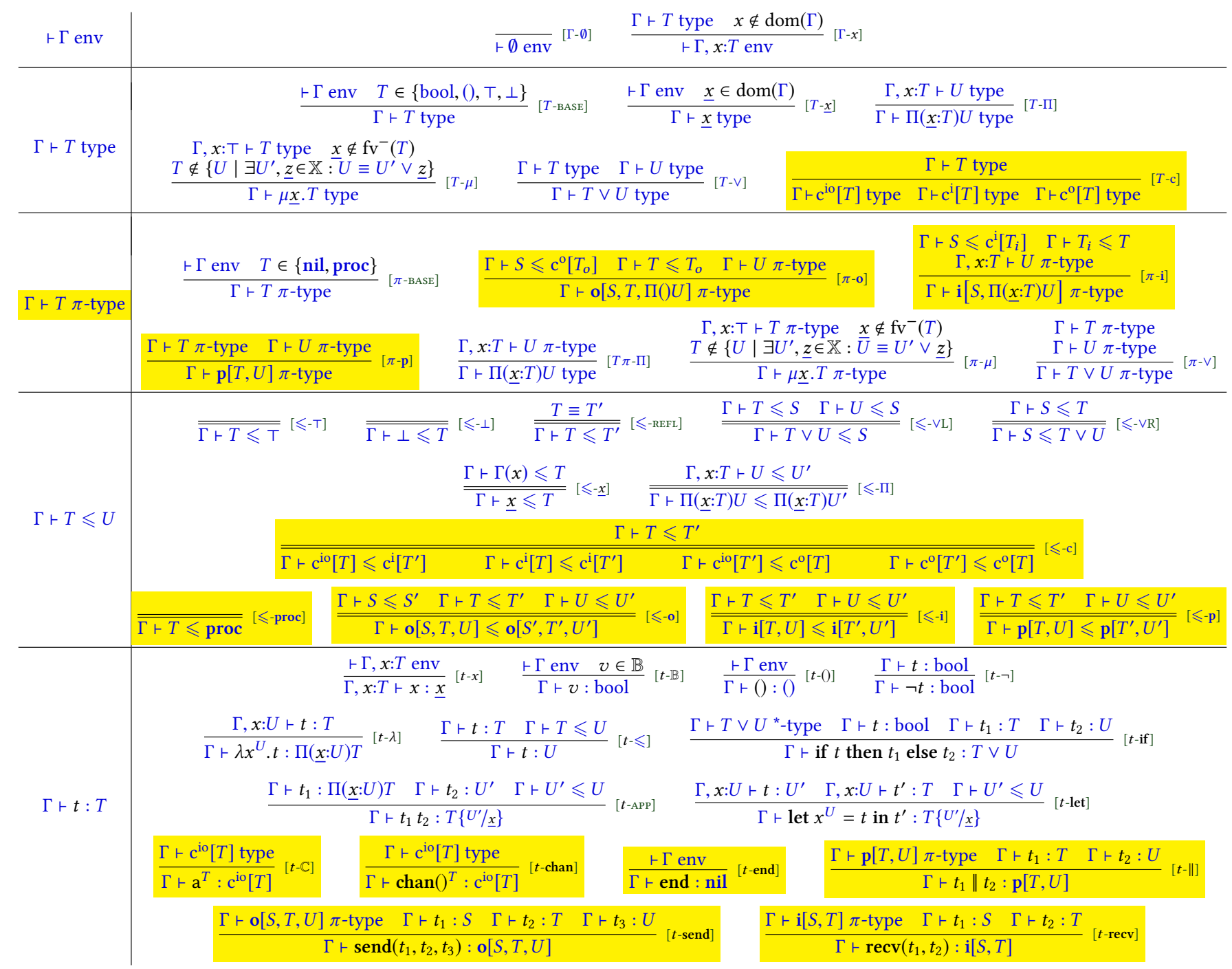

Figure 4. Judgements of the $\lambda_{\leqslant}^{\pi}$ type system (Def. 3.2). The main concurrency-related rules are highlighted.

$T_{\text {ping }} y \underline{z}$ and $T_{\text {pong }} \underline{z}$ (yielded by rule [t-App], Fig. 4) substitute $\bar{y}$ and $\underline{z}$ in $T_{\text {ping }}$ and $T_{\text {pong }}$ 's bodies (by Def. 3.1). This is obtained by leveraging dependent function types, and is key for combining types/protocols and verifying them (§4).

Example 3.4 (Mobile code). Modern languages and toolkits for message-passing programs support sending/receiving mobile code (e.g., [18, 49, 52]). Consider this scenario: a data analysis server lets its clients send custom code, for on-thefly data filtering. In $\lambda \underset{\leqslant}{\pi}$, the intended behaviour of custom code can be formalised by a type like $T_{m}$ below: it describes an abstract process, taking two input channels $\underline{i_{1}} / \underline{i_{2}}$, and an output channel $\underline{o}$; it must use $\underline{i}_{1} / \underline{i}_{2}$ to input integers $\underline{x} / y$, and then it must send one of them along $\underline{o}$, recursively.

$$
\begin{aligned}
T_{m}=\Pi\left(\underline{i_{1}}: \mathrm{c}^{\mathrm{i}}[\mathrm{int}]\right) \Pi\left(\underline{i_{2}}: \mathrm{c}^{\mathrm{i}}[\mathrm{int}]\right) \Pi\left(\underline{o}: \mathrm{c}^{\mathrm{o}}[\mathrm{int}]\right) \\
\mu \mathrm{t} . \mathrm{i}\left[\underline{i_{1}}, \Pi(\underline{x}: \text { int }) \mathrm{i}\left[\underline{i_{2}}, \Pi(\underline{y}: \text { int }) \mathbf{o}[\underline{o},(\underline{x} \vee \underline{y}), \Pi() \mathrm{t}]\right]\right]
\end{aligned}
$$

By inspecting $T_{m}$, we infer that, e.g., $T_{m}$-typed terms cannot be forkbombs; also, " $\underline{x} \vee y$ " does not allow to send on out a value not coming from $\overline{i_{1}} / i_{2}$ (we will formalise these intuitions in Ex. 4.11). The terms below implement $T_{m}: m_{1}$ always sends $x$ received from $i_{1}$, then recursively calls itself, swapping $i_{1} / i_{2} ; m_{2}$ sends the maximum between $x$ and $y$.

$$
\begin{aligned}
& \text { let } m_{1}=\lambda i_{1} \cdot \lambda i_{2} \cdot \lambda o \text {. } \\
& \operatorname{recv}\left(i_{1}, \lambda x \cdot \operatorname{recv}\left(i_{2}, \lambda_{-} \cdot \operatorname{send}\left(o, x, \lambda_{-} . m_{1} i_{2} i_{1} o\right)\right)\right) \\
& \text { let } m_{2}=\lambda i_{1} \cdot \lambda i_{2} \cdot \lambda o \text {. } \\
& \operatorname{recv}\left(i_{1}, \lambda x \cdot \operatorname{recv}\left(i_{2}, \lambda y \text {. }\right)\right. \\
& \left.\operatorname{send}\left(o,(\text { if } x>y \text { then } x \text { else } y), \lambda_{-} . m_{2} i_{1} i_{2} o\right)\right)
\end{aligned}
$$

Below, srv is a data processing server. It takes two channels: $\mathrm{cm}$ and out; it creates two private channels $z_{1}$ and $z_{2}$, uses $\mathrm{cm}$ to receive an abstract process $p$, and runs it, in parallel with two producers (omitted) that send values on $z_{1} / z_{2}$ : 


$$
\begin{aligned}
& \text { let } \operatorname{srv}=\lambda c m . \lambda \text { out. } \\
& \text { let } z_{1}=\operatorname{chan}() \text { in let } z_{2}=\operatorname{chan}() \text { in } \\
& \quad \operatorname{recv}\left(c m, \lambda p \cdot\left(p z_{1} z_{2} \text { out }\left\|\operatorname{prod}_{1} z_{1}\right\| \operatorname{prod}_{2} z_{2}\right)\right)
\end{aligned}
$$

The system works correctly if the received code $p$ is $m_{1}$ or $m_{2}$ above - or any instance of $T_{m}$. To ensure that $s r v$ can only receive a $T_{m}$-typed term on $\mathrm{cm}$, we check its type:

$$
\emptyset \vdash s r v: T_{s r v}=\Pi\left(\underline{\mathrm{cm}}: \mathrm{c}^{\mathrm{i}}\left[T_{m}\right]\right) \Pi\left(\underline{\text { out }}: \mathrm{c}^{\mathrm{o}}[\mathrm{int}]\right) \text { proc }
$$

and this guarantees that, e.g., the parallel composition

$$
\operatorname{send}\left(x, t, \lambda_{-} \text {.end) } \| \operatorname{srv} x \text { out (client sends } t \text { to server, via } x\right. \text { ) }
$$

is typable in $\Gamma$ only if $\Gamma \vdash x: \mathrm{c}^{\mathrm{io}}\left[T_{m}\right]$, implying $\Gamma \vdash t: T_{m}$. We can replace proc with a more precise type. If $U_{1} / U_{2}$ are types of $\operatorname{prod}_{1} / \operatorname{prod}_{2}$, the $\operatorname{recv}(\ldots)$ sub-term of $s r v$ has type:

$$
T_{s r v}^{\prime}=\mathbf{i}\left[\underline{c m}, \Pi\left(\underline{p}: T_{m}\right) \mathbf{p}\left[\mathbf{p}\left[T_{m} \underline{z_{1}} \underline{z_{2}} \underline{\text { out }}, U_{1} \underline{z_{1}}\right], U_{2} \underline{z_{2}}\right]\right]
$$

i.e., the server uses $\mathrm{cm}$ to receive a $T_{m}$-typed abstract process $p$, and then behaves as $T_{m}$ (applied to $z_{1}, z_{2}$, out) composed in parallel with $U_{1} / U_{2}$ (applied to $z_{1} / z_{2}$ ).

Subtyping, subsumption, and private channels The subtyping rules in Fig. 4 are standard (based on $F_{<:}[8,32]$ ) except the highlighted ones. By rule [ $\leqslant$-c], subtyping for channel types is covariant for inputs, and contravariant for outputs, as expected [61]: intuitively, channels with smaller types can

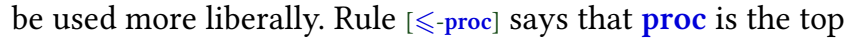
type for $\pi$-types. Rules $[\leqslant-\mathbf{o}] /[\leqslant-\mathbf{i}] /[\leqslant-$ p] say that types for input/output/parallel processes are covariant in all parameters.

As usual, supertyping / subsumption (rule $[t-\leqslant]$ ) caters for Liskov \& Wing's substitution principle [51]: a smaller object can replace a larger one. Crucially, in our theory, supertyping also allows to drop information when typing private channels. This is shown in Ex. 3.5: via supertyping, we do not precisely track how private (i.e., bound) channels are used. This information loss is key to type Turing-powerful $\lambda_{\leqslant}^{\pi}$ terms with a non-Turing-complete type language, for the results in $\S 4$.

Example 3.5 (Subtyping, binding, and precision loss). Let:

$$
\begin{aligned}
t_{1} & =\operatorname{send}\left(x, 42, \lambda_{-} \text {.end }\right) \| \operatorname{recv}\left(x, \lambda_{-} . \text {end }\right) \\
t_{2} & =\left(\text { let } z=\operatorname{chan}() \text { in } \operatorname{send}\left(z, 42, \lambda_{-} . \text {end }\right)\right) \| \operatorname{recv}\left(x, \lambda_{-} . \text {end }\right) \\
T_{1} & =\mathbf{p}[\mathbf{o}[\underline{x}, \text { int }, \Pi() \text { nil }], \mathrm{i}[\underline{x}, \Pi(\underline{y}: \text { int }) \text { nil }]] \\
T_{2} & =\mathbf{p}\left[\mathbf{o}\left[\mathrm{c}^{\text {io }}[\text { int }], \text { int, } \Pi() \text { nil }\right], \mathbf{i}[\underline{x}, \Pi(\underline{y} \text { :int }) \text { nil }]\right]
\end{aligned}
$$

Letting $\Gamma=x: \mathrm{c}^{\mathrm{io}}[\mathrm{int}]$, we have $\Gamma \vdash \underline{x} \leqslant \mathrm{c}^{\mathrm{io}}[$ int $]$ and $\Gamma \vdash$ $T_{1} \leqslant T_{2}$. For $t_{1}$, we have both $\Gamma \vdash t_{1}: T_{1}$ and $\Gamma \vdash t_{1}: T_{2}$ (by $[t-\leqslant])$ : in the first judgement, $T_{1}$ precisely captures that $x$ is used to send/receive an integer; instead, in the second judgement, $T_{2}$ is less accurate, and says that some term with type $c^{\text {io }}$ [int] is used to send, while $x$ is used to receive.

We also have $\Gamma \vdash t_{2}: T_{2}$; and notably, since $z$ is bound in the "let..." subterm of $t_{2}$, it cannot appear in the type: i.e., we cannot write a more accurate type for $t_{2}$. This is due to rule [t-let] (Fig. 4): since $z$ is bound by let..., its occurrence in $\operatorname{send}(\ldots)$ is typed by a supertype of $\underline{z}$ that is suitable for both $z$ and $\operatorname{chan}()-$ in this case, $\mathrm{c}^{\mathrm{io}}[\mathrm{int}]$. Specifically:

$$
\begin{aligned}
& \Gamma \vdash \mathrm{c}^{\mathrm{io}}[\text { int }] \leqslant \mathrm{c}^{\mathrm{io}}[\text { int }] \quad \Gamma, z: \mathrm{c}^{\mathrm{io}}[\text { int }] \vdash \operatorname{chan}(): \mathrm{c}^{\mathrm{io}}[\text { int }] \\
& \quad \Gamma, z: \mathrm{c}^{\mathrm{io}}[\text { int }] \vdash \operatorname{send}\left(z, 42, \lambda_{-} . \text {end }\right): \mathrm{o}[\underline{z}, \text { int, } \Pi() \text { nil }] \quad[t \text {-let }] \\
& \hline \Gamma \vdash \text { let } z=\operatorname{chan}() \text { in send }\left(z, 42, \lambda_{-} . \text {end }\right): \mathrm{o}[\underline{z}, \text { int }, \Pi() \text { nil }]\left\{\mathrm{c}^{\mathrm{i} o}[\text { int }] / \underline{z}\right\}
\end{aligned}
$$

Typing guarantees that well-typed terms never go wrong.

Theorem 3.6 (Type safety). If $\Gamma \vdash t: T$, then $t$ is safe.

Thm. 3.6 follows by: $\Gamma \vdash t: T$ and $t \rightarrow t^{\prime}$ implies $\exists T^{\prime}$ such that $\Gamma \vdash t^{\prime}: T^{\prime}-$ i.e., typed terms only reduce to typed terms, without (untypable) err subterms. In $§ 4$, we study how $T$ and $T^{\prime}$ are related, and how they constraint $t$ 's behaviour.

\section{Type-Level Model Checking}

Our typing discipline guarantees conformance between processes and types (Fig. 4), and absence of run-time errors (Thm. 3.6). However, as seen in $§ 1$, our types can describe a wide range of behaviours, from desirable ones (e.g., formalising a specification), to undesirable ones (e.g., deadlocks); moreover, complex (and potentially unwanted) behaviours can arise when $\lambda \leqslant$ terms are allowed to interact.

To avoid this issue, we might want to check whether a process $t$ (possibly consisting of multiple parallel sub-processes) satisfies a property $\phi$ in some temporal logic [73]: $\phi$ could be, e.g., a safety property $\square\left(\neg \phi^{\prime}\right)$ (“ $\phi^{\prime}$ is never true while $t$ runs") or a liveness property $\diamond \phi^{\prime}$ (" $t$ will eventually satisfy $\phi^{\prime \prime}$ ). However, this problem is undecidable (unless $\phi$ is trivial), since $\lambda_{\leqslant}^{\pi}$ is Turing-powerful even in its productive fragment (due to recursion and channel creation [7]).

Luckily, our theory allows to: (1) mimic the parallel composition of terms by composing their types (as shown in Ex. 3.3), and (2) mimic the behaviour of processes by giving a semantics to types (as we show in this section). This means that we can ensure that a (composition of) typed process(es) $t$ has a desired safety/liveness property, by model-checking its type $T$ (that is not Turing-powerful). Moreover, we do not need to know how $t$ is implemented: we only need to know that it has type $T$. We now illustrate the approach, and its preconditions (roughly: for the verification of liveness properties, we need productivity, and use of open variables).

Outline First, we need to surmount a typical obstacle for behavioural type systems. Ex. 3.5 shows that accurate types require open terms in their typing environment - but Def. 2.4 works on closed terms; so, observing how $T_{1}$ in Ex. 3.5 uses $\underline{x}$, we sense that $t_{1}$ should interact via $x$ - but by Def. 2.4, $t_{1}$ is stuck. To trigger communication, we may bind $x$ in $t_{1}$ with a channel instance, e.g., $t_{1}^{\prime}=$ let $x=\operatorname{chan}()$ in $t_{1}-$ but $t_{1}^{\prime}$ 's type cannot mention $\underline{x}$, hence cannot convey which channel(s) $t_{1}^{\prime}$ uses. Thus, we develop a type-based analysis in four steps: (1) we define an over-approximating LTS semantics for typed $\lambda \leqslant$ terms with free variables (Def. 4.1); (2) we define an LTS semantics for types (Def. 4.2); (3) we prove subject transition and type fidelity (Thm. 4.4, 4.5); (4) using them, we show how temporal logic judgements on types transfer to processes. 
Definition 4.1 (Labelled semantics of open typed terms). When $\Gamma \vdash t: T$ (for any $\Gamma, t, T$ ), the judgements $\Gamma \vdash t \stackrel{\alpha}{\rightarrow} t^{\prime}$ and $\Gamma \vdash t \stackrel{\tau^{\bullet}}{\longrightarrow} t^{\prime}$ are inductively defined in Fig. 5.

Unlike Def. 2.4, Def. 4.1 lets an open term like $\neg x$ reduce, by non-deterministically instantiating $x$ to tt or ff; the assumption $\Gamma \vdash \neg x: T$ ensures that $x$ is a boolean. Rule [SR- $\rightarrow$ ] inherits "concrete" reductions from Def. 2.4: if $t \rightarrow t^{\prime}$ is induced by base rule $[\mathrm{R}]$, the transition label is $\tau[\mathrm{r}]$. Rules [SR-send]/[SR-recv] send/receive a value/variable $w^{\prime}$ using a (channel-typed) value/variable $w$. Note that in [SRrecv], $w^{\prime}$ is any value/variable of type $T_{i}$, which is the input type of $x$ (in $\pi$-calculus jargon, it is an early semantics [63]). Rule [SR-Сомм] lets processes exchange a payload $w^{\prime}$ via a channel/variable $w$, recording $w$ in the transition label. Rule [SR- $x(0)$ "applies" $x$ by instantiating it with any suitablytyped $\lambda y . v$ (i.e., $\lambda y . v$ must be a function that, when applied to $w$, yields a term $v\{w / y\}$ of type $T$ ); it also records $x$ in the transition label. Rule [SR- $\lambda(0)$ applies a function to a variable $x$, with the expected substitution. Rule [SR-E] propagates transitions through contexts, unless labels refer to bound variables. Finally, $\Gamma \vdash t \stackrel{\tau^{*}}{\longrightarrow} * t^{\prime}$ holds when $t$ reaches $t^{\prime}$ via a finite sequence of internal moves excluding interaction: i.e., labels $w\left(w^{\prime}\right), \bar{w}\left\langle w^{\prime}\right\rangle, \tau[w]$, and $\tau$ [R-Сомм] are forbidden.

Using Def. 4.1 on $t_{1}$ from Ex. 3.5, we get the transition $\Gamma \vdash t_{1} \stackrel{\tau[x]}{\longrightarrow}$ end $\|$ end, and we observe the use of $x$, as desired.

Type semantics We now equip our types with labelled transition semantics (Def. 4.2): this is not unusual for behavioural type systems in $\pi$-calculus literature $[3,30]$ - but our novel use of type variables, and dependent function types, yields new capabilities, and requires some sophistication.

The type transitions should mimic the semantics of typed processes. Hence, take $T_{1}$ and $t_{1}$ from Ex. 3.5: we want $T_{1}$ to

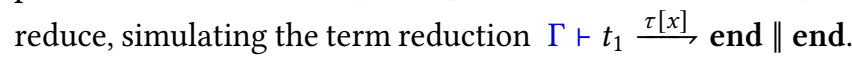
This suggests that a type like $\mathrm{p}[\mathrm{o}[\underline{x}, \ldots], \mathrm{i}[\underline{x}, \ldots]]$ should reduce with a communication on $\underline{x}$. But consider $T_{2}$ in Ex. 3.5: $T_{2}$ also types $t_{1}$, hence it should also simulate $t_{1}$ 's reduction i.e., a type like $\mathrm{p}\left[\mathrm{o}\left[\mathrm{c}^{\mathrm{i} o}[\mathrm{int}], \ldots\right], \mathrm{i}[\underline{x}, \ldots]\right]$ should reduce, too. In general, we want $\mathrm{p}[\mathrm{o}[S, \ldots], \mathrm{i}[T, \ldots]]$ to reduce if $S$ and $T$ "might interact", i.e., they could type a same channel/variable: we formalise this idea as $\Gamma \vdash S \bowtie T$ in Def. 4.2.

Definition 4.2 (Type semantics). Let $S \sqcap_{\Gamma} T$ be the greatest subtype of $S$ and $T$ in $\Gamma$, up-to $\equiv$ (Def. 3.1). The judgement $\Gamma \vdash S \bowtie T$ (read " $S$ and $T$ might interact in $\Gamma$ ") is:

$$
\frac{\Gamma \nvdash S \sqcap_{\Gamma} T \leqslant \perp}{\Gamma \vdash S \bowtie T}[\bowtie \triangleleft-\mathrm{c}]
$$

A type reduction context $\mathcal{E}$ is inductively defined as:

[ ] $|\mathbf{o}[\mathcal{E}, T, U]| \mathbf{o}[S, \mathcal{E}, U]|\mathbf{o}[S, T, \mathcal{E}]| \mathbf{i}[\mathcal{E}, T]|\mathrm{i}[S, \mathcal{E}]| \mathbf{p}[\mathcal{E}, T]$ Judgements $\Gamma \vdash T \stackrel{\alpha}{\rightarrow} T^{\prime}$ and $\Gamma \vdash T \stackrel{\tau[\mathrm{v}]}{\longrightarrow} T^{\prime}$ are in Fig. 6 .

By Def. 4.2, $\Gamma \vdash S \bowtie S^{\prime}$ holds when $S$ and $S^{\prime}$ have a common subtype besides $\perp$, i.e., they might type a same term in $\Gamma$, via rule $[t-\leqslant]$. The judgement $\Gamma \vdash T \stackrel{\alpha}{\rightarrow} T^{\prime}$ says that $T \vee U$ can reduce to $T$ or $U$, firing label $\tau[\mathrm{V}]$. Rule $[T \rightarrow \mathrm{o}]$ reduces an output type, recording the used channel type $S$ and payload $T$ in the transition label. Rule $[T \rightarrow \mathrm{i}]$ is similar for input types, recording the payload $T^{\prime}$. We have two communication rules:

- $\left[T \rightarrow\right.$ iox $x$ fires when, in $\mathbf{p}\left[U, U^{\prime}\right]$, there might be an interaction with a type variable $\underline{x}$ as payload. Note that, by $[T \rightarrow \mathrm{i}]$, the $x$ sent by $U$ is substituted in $U^{\prime \prime \prime}$, hence it can appear in its future transitions. The rule yields a transition label $\tau\left[S, S^{\prime}\right]$, recording which channel types were used;

- $[T \rightarrow$ io $]$ is similar, but fires if the payload $T$ is not a variable. Finally, $\Gamma \vdash T \stackrel{\tau[\mathrm{v}]}{\longrightarrow} T^{\prime}$ holds if $T$ reaches $T^{\prime}$ via a finite sequence of internal choices $\tau[\mathrm{V}]$.

Example 4.3. Take sys from Ex. 2.2, $T_{p p}$ from Ex. 3.3. Let:

$\Gamma=y: \mathrm{c}^{\mathrm{io}}[\operatorname{str}], z: \mathrm{c}^{\mathrm{io}}\left[\mathrm{c}^{\mathrm{o}}[\mathrm{str}]\right]$

$\begin{aligned} t & =\text { sys } y z \\ T & =T_{p p} \underline{y} \underline{z}\end{aligned}=\mathrm{p}\left[\begin{array}{l}\mathrm{o}[\underline{z}, \underline{y}, \mathrm{i}[\underline{y}, \Pi(\text { reply:str)nil }]], \\ \mathrm{i}\left[\underline{z}, \Pi\left(\underline{\left.\text { replyTo: } \mathrm{c}^{\mathrm{o}}[\mathrm{str}]\right) \mathrm{o}}[\underline{\text { replyTo, str, } \Pi() \mathrm{nil}]}]\right]\right.\end{array}\right]$

By Def. 3.2, we have $\Gamma \vdash t: T$. By Def. 4.1, we have:

$$
\Gamma \vdash t \stackrel{\tau[z]}{\underset{\tau^{\bullet}}{\longrightarrow}} *\left(\begin{array}{l}
\operatorname{recv}(y, \ldots) \| \\
\operatorname{send}(y, " \mathrm{Hi} ! ", \ldots)
\end{array}\right) \stackrel{\tau[y]}{\longrightarrow} \stackrel{\tau^{\bullet}}{\longrightarrow} *\left(\begin{array}{l}
\text { end } \| \\
\text { end }
\end{array}\right)
$$

By Def. 4.2, applying rule $[T \rightarrow$ iox $]$ twice, we get:

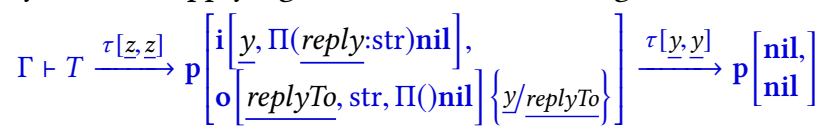

Observe that $T$ closely mimicks the transitions of $t$ : the typelevel substitution of $y$ in place of replyTo allows to track the usage of $y$ after its transmission, capturing ponger's reply to pinger. This realises our insight: tracking inputs/outputs of programs, by using variables in their types. Technically, it is achieved via the dependent function type inside i $[\ldots, \ldots]$.

Subject transition and type fidelity With the semantics of Def. 4.1, we prove a result yielding Thm. 3.6 as a corollary.

Theorem 4.4 (Subject transition). Assume $\Gamma \vdash t: T$. If $\Gamma \vdash T$ type, then $\Gamma \vdash t \stackrel{\alpha}{\rightarrow} t^{\prime}$ implies $\Gamma \vdash t^{\prime}: T$. Otherwise, when $\Gamma \vdash T \pi$-type, we have:

1. $\Gamma \vdash t \stackrel{\alpha}{\rightarrow} t^{\prime}$ with $\tau^{\bullet}(\alpha)$ (Fig. 5) implies $\Gamma \vdash t^{\prime}: T$;

2. $\Gamma \vdash t \stackrel{\alpha}{\sim} t^{\prime}$ and $\alpha \in\{\bar{x}\langle w\rangle, x(w), \tau[x], \tau[R$-Сомм $]\}$ implies one:

a. $\Gamma \vdash t^{\prime}: T$ and proc $\in T$;

b. $\alpha=\bar{x}\langle w\rangle$ and $\exists S, U, T^{\prime}: \Gamma \vdash x: S, w: U, t^{\prime}: T^{\prime}$ and $\Gamma \vdash T \stackrel{\tau[\mathrm{V}]}{\longrightarrow} * \stackrel{\bar{s}\langle U\rangle}{\longrightarrow} T^{\prime} ;$

c. $\alpha=x(w)$ and $\exists S, U, T^{\prime}: \Gamma \vdash x: S, w: U, t^{\prime}: T^{\prime}$ and $\Gamma \vdash T \stackrel{\tau[\mathrm{v}]}{\longrightarrow} * \stackrel{S(U)}{\longrightarrow} T^{\prime}$

d. $\alpha=\tau[x]$ and $\exists S, S^{\prime}, T^{\prime}: \Gamma \vdash x: S, x: S^{\prime}, t^{\prime}: T^{\prime}$ and $\Gamma \vdash T \stackrel{\tau[\mathrm{V}]}{\longrightarrow} * \stackrel{\tau\left[S, S^{\prime}\right]}{\longrightarrow} T^{\prime}$

e. $\alpha=\tau[R$-сомм $]$ and $\exists S, S^{\prime}, T^{\prime}:\left\{S, S^{\prime}\right\} \nsubseteq \mathbb{X}, \Gamma \vdash t^{\prime}: T^{\prime}$ and $\Gamma \vdash T \stackrel{\tau[\mathrm{v}]}{\longrightarrow} * \frac{\tau\left[S, S^{\prime}\right]}{\longrightarrow} T^{\prime}$

Assume $\Gamma \vdash t: T$, with $t$ reducing to $t^{\prime}:$ Thm 4.4 says that when the reduction is caused by the functional fragment of $\lambda_{\leqslant}^{\pi}$ (hypothesis $\Gamma \vdash T$ type, or case 1 ), then $t^{\prime}$ has the same 


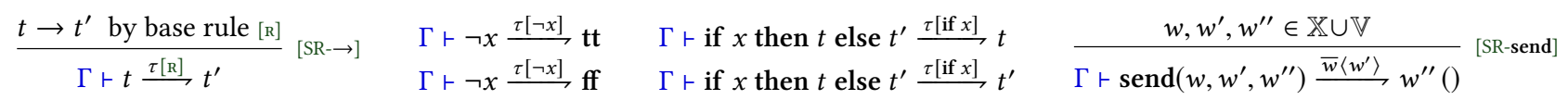

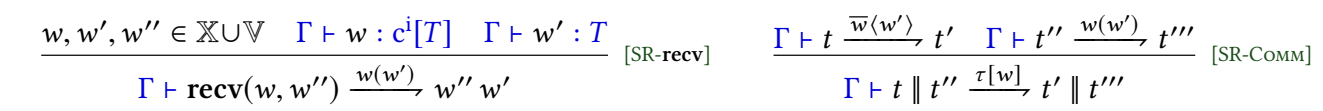

$$
\begin{aligned}
& \frac{\Gamma \vdash x w: T \quad w \in \mathbb{X} \cup \mathbb{V} \quad \Gamma \vdash v\{w / y\}: T}{\Gamma \vdash x w \stackrel{\tau[x()]}{\longrightarrow} v\{w / y\}}[\mathrm{SR}-x()] \quad \frac{\Gamma+t^{\prime} \stackrel{\alpha}{\rightarrow} t^{\prime \prime} \operatorname{fv}(\alpha) \cap \operatorname{bv}(\mathcal{E})=\emptyset}{\Gamma \vdash \mathcal{E}[t] \frac{\alpha}{\longrightarrow} \mathcal{E}\left[t^{\prime}\right]}[\mathrm{SR}-\mathcal{E}]
\end{aligned}
$$

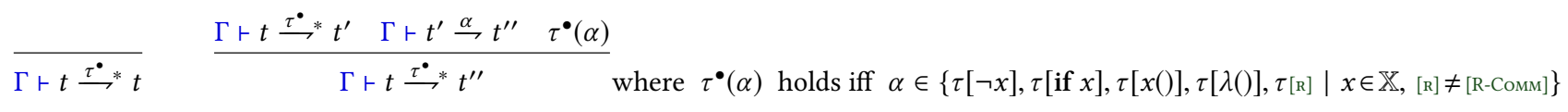

Figure 5. Over-approximating labelled semantics of $\lambda_{\leqslant}^{\pi}$ terms. We will sometimes use label $\tau$ to denote any $\tau[\cdot]$-label above.

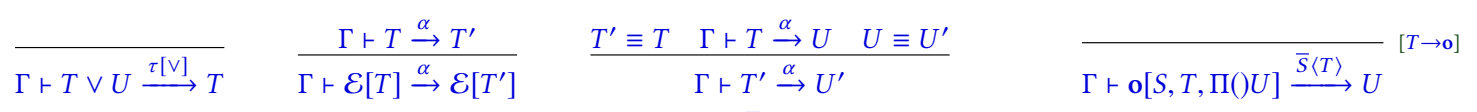

$$
\begin{aligned}
& \frac{\Gamma \vdash T^{\prime} \leqslant T \quad T^{\prime}=T \text { or } T^{\prime} \in \mathbb{X}}{\Gamma \vdash \mathrm{i}[S, \Pi(\underline{x}: T) U] \stackrel{S\left(T^{\prime}\right)}{\longrightarrow} U\left\{T^{\prime} / \underline{x}\right\}}[T \rightarrow \mathrm{i}] \quad \frac{\Gamma \vdash U \stackrel{\bar{S}\langle\underline{x}\rangle}{\longrightarrow} U^{\prime} \quad \Gamma \vdash U^{\prime \prime} \stackrel{S^{\prime}(\underline{x})}{\longrightarrow} U^{\prime \prime \prime} \quad \Gamma \vdash S \bowtie S^{\prime}}{\Gamma \vdash \mathrm{p}\left[U, U^{\prime \prime}\right] \stackrel{\tau\left[S, S^{\prime}\right]}{\longrightarrow} \mathrm{p}\left[U^{\prime}, U^{\prime \prime \prime}\right]}[T \rightarrow \mathrm{iox}]
\end{aligned}
$$

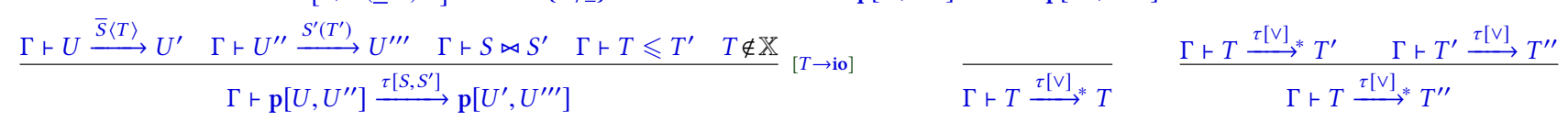

Figure 6. Semantics of $\lambda \underset{\leqslant}{\pi}$ types. We will sometimes use label $\tau$ to denote either $\tau[\vee]$ or $\tau\left[S, S^{\prime}\right]$ (for some $S, S^{\prime}$ ).

type $T$. Instead, if the reduction is caused by input, output or interaction events, then we observe a corresponding labelled transition in the type, possibly after some $\tau[\mathrm{V}]$ moves (cases $2 \mathrm{~b}-2 \mathrm{e}$ ); the exception is case $2 \mathrm{a}$ : if $t^{\prime}$ keeps type $T$, then that $T$ syntactically contains proc, which types a reducing sub-term of $t$ before and after its reduction (via rule $[t-\leqslant]$ ).

We can also prove the opposite direction of Thm. 4.4: if type $T$ interacts, then a typed term $t$ interacts accordingly. This intuition holds under two conditions, leading to Thm. 4.5:

(c1) we only use productive $\lambda_{\leqslant}^{\pi}$ terms, i.e., all functions must be total (always return a value or process when applied). This means that, e.g., if $\Gamma \vdash t: \mathbf{o}\left[\underline{x}\right.$, int, $\left.T^{\prime}\right]$, then $t$ will output on $x$; this excludes cases like $t=$ if $\omega$ then $\operatorname{send}\left(x, 42, t^{\prime}\right)$ else $\operatorname{send}\left(x, 43, t^{\prime \prime}\right)$ (with $\omega=$ $(\lambda y \cdot y y)(\lambda z \cdot z z))$. Productivity is obtained with many methods from literature (e.g., [21, 72]);

(c2) the subjects of input/output/interaction transitions of $T$ must be type variables: this allows to precisely relate them to occurrences of (open) variables in $t$.

Theorem 4.5 (Type fidelity). Within productive $\lambda_{\leqslant}^{\pi}$, assume $\Gamma \vdash t: T$ and $\Gamma \vdash T \pi$-type. Then:

1. $\Gamma \vdash T \stackrel{\bar{x}\langle U\rangle}{\longrightarrow} T^{\prime}$ implies $\exists w, t^{\prime}: \Gamma \vdash w: U, t^{\prime}: T^{\prime}$ and $\Gamma \vdash t \stackrel{\tau^{\bullet}}{\longrightarrow}(U) \stackrel{\bar{x}\langle w\rangle}{\longrightarrow} t^{\prime}$

2. $\Gamma \vdash T \stackrel{\underline{x}(U)}{\longrightarrow} T^{\prime}$ implies $\forall w:$ if $\Gamma \vdash w: U$, then $\exists t^{\prime}$ : $\Gamma \vdash t^{\prime}: T^{\prime}$ and $\Gamma \vdash t \stackrel{\tau^{\bullet}}{\longrightarrow} \stackrel{x(w)}{\longrightarrow} t^{\prime}$;

3. $\Gamma \vdash T \stackrel{\tau[\underline{x}, \underline{x}]}{\longrightarrow} T^{\prime}$ implies $\exists t^{\prime}$ such that $\Gamma \vdash t^{\prime}: T^{\prime}$ and $\Gamma \vdash t \stackrel{\tau^{\bullet}}{\longrightarrow} \stackrel{\tau[x]}{\longrightarrow} t^{\prime}$
4. $\Gamma \vdash T \stackrel{\tau[\mathrm{V}]}{\longrightarrow}$ implies either: (a) $\exists T^{\prime}: \Gamma \vdash T \stackrel{\tau[\mathrm{V}]}{\longrightarrow} T^{\prime}$ and $\Gamma \vdash t: T^{\prime} ;$ or, (b) $\exists t^{\prime}: \Gamma \vdash t \stackrel{\alpha}{\rightarrow} t^{\prime}$ with $\tau^{\bullet}(\alpha)$ (Fig. 5) and $\Gamma \vdash t^{\prime}: T$; or, (c) $\exists T^{\prime}: \Gamma \vdash T \stackrel{\alpha}{\rightarrow} T^{\prime}$ with $\alpha \neq \tau[\mathrm{\vee}]$.

Items 1-3 of Thm. 4.5 say that if $T$ can input/output/interact, then $t$ can do the same, possibly after a sequence of $\tau$-steps (without communication, cf. Def. 4.1); the $\tau$-sequence is finite, since $t$ is productive by hypothesis. By item 4 , if $T$ can make a choice $(\mathrm{V})$, then $t$ could have already chosen one option (case (a)), or could choose later (cases (b) or (c)).

Process verification via type verification By exploiting the correspondence between process / type reductions in Thm. 4.4 and 4.5, we can transfer (decidable) verification results from types to processes. To this purpose, we analyse the labelled transition systems (LTSs) of types and processes using the linear-time $\mu$-calculus [20, §3]. We chose it for two reasons: (1) the open term/type semantics (Def. 4.1/4.2) are over-approximating, and a linear-time logic is a natural tool to ensure that all possible executions ("real" or approximated) satisfy a formula; and (2) linear-time $\mu$-calculus is decidable for our types, with minimal restrictions (Lemma 4.7).

Definition 4.6 (Linear-time $\mu$-calculus). Given a set of actions $\mathbb{A}$ ct ranged over by $\alpha$, the linear-time $\mu$-calculus formulas are defined as follows (where $\mathbb{A}$ is a subset of $\mathbb{A c t}$ ):

Basic formulas: $\phi::=\mathrm{Z}|\neg \phi| \phi_{1} \wedge \phi_{2}|(\alpha) \phi| v \mathrm{Z} . \phi$ Derived $\left\{\top|\perp| \phi_{1} \vee \phi_{2}\left|\phi_{1} \Rightarrow \phi_{2}\right| \mu \mathrm{Z} . \phi\right.$ formulas $\left\{(\mathbb{A}) \phi|(-\mathbb{A}) \phi| \phi_{1} \cup \phi_{2}|\square \phi| \diamond \phi\right.$

In Def. 4.6, $\phi$ describes accepted sequences of actions; $\phi$ can be a variable $Z$, negation, conjunction, prefixing $(\alpha) \phi$ 
("accept a sequence if it starts with $\alpha$, and then $\phi$ holds"), or greatest fixed point $v Z$. $\phi$. Basic formulas are enough $[6,73]$ to derive true/false (accept any/no sequence of actions), disjunction, implication, least fixed points $\mu \mathrm{Z} . \phi ;(\mathbb{A}) \phi$ accepts sequences that start with any $\alpha \in \mathbb{A}$, then satisfy $\phi$; dually, $(-\mathbb{A}) \phi$ requires $\alpha \in \mathbb{A} c t \backslash \mathbb{A}$. We also derive usual temporal formulas $\phi_{1} \cup \phi_{2}$ (“ $\phi_{1}$ holds, until $\phi_{2}$ eventually holds"), $\square \phi$ (" $\phi$ is always true"), and $\nabla \phi$ (“ $\phi$ is eventually true"). Given a process $p$ with LTS of labels $\mathbb{A c t}$, a run of $p$ is a finite or infinite sequence of labels fired along a complete execution of $p$; we write $p=\phi$ if $\phi$ accepts all runs of $p$. (Details: [70])

We can decide $\phi$ on a guarded type T, as shown in Lemma 4.7. Here, we instantiate $\mathbb{A}$ ct (Def. 4.6) as $\mathbb{A}_{\Gamma}(T)$, which is the set of labels fired along $T$ 's transitions in $\Gamma$, (Def. 4.2); notably, $\mathbb{A}_{\Gamma}(T)$ is finite and syntactically determined. (Details: [70])

Lemma 4.7. Given $\Gamma$, we say that $T$ is guarded iff, for all $\pi$-type subterms $\mu \mathrm{t}$. $U$ of $T, \mathbf{t}$ can occur in $U$ only as subterm of $\mathrm{i}[\ldots]$ or $\mathrm{o}[\ldots]$; then, if $T$ is guarded, $T=\phi$ is decidable.

Lemma 4.7 holds since guarded $\pi$-types are encodable in CCS without restriction [53], then in Petri nets [22, §4.1], for which linear-time $\mu$-calculus is decidable [20]. Notably, Lemma 4.7 covers infinite-state types (with $\mathrm{p}[\ldots, \ldots]$ under $\mu \mathrm{t} . .$. ), that type $\lambda_{\leqslant}^{\pi}$ terms with unbounded parallel subterms.

Now, assuming $\Gamma \vdash t: T$, we can ensure that $\phi$ holds for $t$, by deciding a related formula $\phi^{\prime}$ on $T$. We need to take into account that type semantics approximate process semantics:

(i1) if we do not want $t$ to perform an action on channel $x$, we check that $T$ never potentially uses type variable $\underline{x}$;

(i2) if we want $t$ to eventually perform an action on channel $x$, we need $t$ productive, and check that $T$ eventually uses $\underline{x}$ - without doing "imprecise" actions before.

We formalise such intuitions in various cases, in Thm. 4.10; but first, we need the tools of Def. 4.8 and 4.9.

Definition 4.8. The input / output uses of $\underline{x}$ by $T$ in $\Gamma$ are: input uses: $\quad \mathbb{U}_{\Gamma, \mathrm{T}}^{\mathrm{i}}(\underline{x})=\left\{S^{\prime}\left(U^{\prime}\right) \in \mathbb{A}_{\Gamma}(T) \mid \Gamma \vdash \underline{x} \leqslant S^{\prime}\right\}$ output uses: $\quad \mathbb{U}_{\Gamma, \mathrm{T}}^{\mathrm{o}}(\underline{x})=\left\{\overline{S^{\prime}}\left\langle U^{\prime}\right\rangle \in \mathbb{A}_{\Gamma}(T) \mid \Gamma \vdash \underline{x} \leqslant S^{\prime}\right\}$

Definition 4.9. Given a set of type (resp. term) variables $\mathbb{Y}$, the $\mathbb{Y}$-limited transitions of $T$ (resp. $t$ ) in $\Gamma$ are:

$$
\begin{gathered}
\frac{\Gamma \vdash T \stackrel{\alpha}{\rightarrow} T^{\prime} \quad \forall S, U: \alpha \in\{S(U), \bar{S}\langle U\rangle\} \text { implies } S \in \mathbb{Y}}{T \uparrow_{\Gamma} \mathbb{Y} \stackrel{\alpha}{\rightarrow} T^{\prime} \uparrow_{\Gamma} \mathbb{Y}} \\
\frac{\Gamma \vdash t \stackrel{\alpha}{\rightarrow} t^{\prime} \quad \forall w, w^{\prime}: \alpha \in\left\{w\left(w^{\prime}\right), \bar{w}\left\langle w^{\prime}\right\rangle\right\} \text { implies } w \in \mathbb{Y}}{t \uparrow_{\Gamma} \mathbb{Y} \stackrel{\alpha}{\longrightarrow} t^{\prime} \uparrow_{\Gamma} \mathbb{Y}}
\end{gathered}
$$

Theorem 4.10. Within productive $\lambda_{\leqslant}^{\pi}$, assume $\Gamma \vdash t: T$, with $\Gamma \vdash T \pi$-type, proc $\notin T$. Also assume, for all $\mathrm{i}\left[S, \Pi(\underline{x}: U) U^{\prime}\right]$ occurring in $T$, that there is $y$ such that $\Gamma \vdash y: U$ holds. ${ }^{1}$

\footnotetext{
${ }^{1}$ This implicitly requires $\Gamma \vdash U$ type, hence $\operatorname{fv}(U) \cap \operatorname{bv}(T)=\emptyset$ : this assumption could be relaxed (with a more complicated clause), but offers a compromise between simplicity and generality, that is sufficient to verify our examples. Besides this, the existence of $y$ such that $\Gamma \vdash y: U$ can
}

For $\mu$-calculus judgements on $T$, let $\mathbb{A} c t=\mathbb{A}_{\Gamma}(T)$, and $\mathbb{A}_{\tau}=$ $\left\{\tau\left[S, S^{\prime}\right] \in \mathbb{A}_{\Gamma}(T) \mid\left\{S, S^{\prime}\right\} \nsubseteq \operatorname{dom}(\Gamma)\right\}$. Then, the implications in Fig. 7 hold.

Assume $\Gamma \vdash t: T$. The sets $\mathbb{U}_{\Gamma, \mathrm{T}}^{\mathrm{i}}(\underline{x}) / \mathbb{U}_{\Gamma, \mathrm{T}}^{\mathrm{o}}(\underline{x})$ in Def. 4.8 contain all transition labels that might be fired by $T$, when $x$ is used for input/output by $t$. The operator $\uparrow_{\Gamma}\left\{x_{i}\right\}_{i \in 1 . . n}$ (Def. 4.9) limits the observable inputs/outputs of $T / t$ to those occurring on channel $x_{i}$ - while other (open) channels can only reduce by communicating, via $\tau$-actions; i.e., $x_{1}, \ldots, x_{n}$ are interfaces to other types/processes, and are "probed" for verification (this is common in model checking tools).

In Thm. 4.10, item (1) can be seen as a case of intuition (i1) above: if $T$ never fires a label $(\square(\neg \ldots))$ that is a potential output use of $x_{i}(i \in 1 . . n)$, then $t$ never uses $x_{i}$ for output. The "potential output use", by Def. 4.8, is any label $\overline{S^{\prime}}\left\langle U^{\prime}\right\rangle$ fired by $T$ where $S^{\prime}$ is a supertype of $\underline{x}$ : this accounts for "imprecise typing", discussed in Ex. 3.5. Item (3) of Thm. 4.10 is a case of intuition (i2): to ensure that $t$ eventually outputs on $x_{i}(i \in 1 . . n)$, we check that $T$ eventually fires a label $\underline{\bar{x}}\langle U\rangle$; moreover, we check $T$ does not fire any label in $\mathbb{A}_{\tau}$, until (U) the output $\underline{\bar{x}}\langle U\rangle$ occurs. The set $\mathbb{A}_{\tau}$ contains all "imprecise" synchronisation labels $\tau\left[S, S^{\prime}\right]$ where either $S$ or $S^{\prime}$ is not a type variable: we exclude them because, if $T$ fires one, then we cannot use Thm. 4.5(3) to ensure that $t$ reduces accordingly; i.e., if we do not exclude $\mathbb{A}_{\tau}$, then $t$ might deadlock and never perform $\overline{x_{i}}\langle w\rangle$ (for any $w$ ). Finally, item (4) combines the intuitions of both previous cases: we want to ensure that whenever $t$ receives $z$ on channel $x$, then it eventually forwards $z$ through channel $y$, without doing other inputs on $x$ before; to this purpose, we check that whenever $T$ inputs $\underline{z}$ on a channel $S$ (representing a potential use of $\underline{x}$ ), then $T$ eventually fires $\bar{y}\langle\underline{z}\rangle$ - without doing potential inputs on $\underline{x}$, nor firing any label in $\mathbb{A}_{\tau}$, before.

Example 4.11. Take $\Gamma, t, T$ in Ex. 4.3. To ensure that $t$ eventually uses $\underline{y}$ to output a message, we check $T \uparrow_{\Gamma}\{\underline{y}\} \mid=\phi$, with $\phi$ in Fig. 7(3) (right).

Take ponger (Ex. 2.2), $T_{\text {pong }}$ (Ex. 3.3), and $\Gamma=z: \mathrm{c}^{\mathrm{i}}\left[\mathrm{c}^{\mathrm{o}}[\mathrm{str}]\right]$. To ensure that the term ponger $z$ is responsive on $z$, we check ( $\left.T_{\text {pong }} \underline{z}\right) \uparrow_{\Gamma}\{\underline{z}\} \models \phi$, with $\phi$ in Fig. 7(6) (right).

Take $T_{s r v}^{\prime}$ (Ex. 3.4). With an easy adaptation of properties (5) and (4) in Fig. 7 (right), we can verify that: in all implementations $s r v^{\prime}$ of $T_{s r v}^{\prime}$, whenever $s r v^{\prime}$ receives any mobile code $p$ (of type $T_{m}$ ) from channel $\mathrm{cm}, s r v^{\prime}$ becomes reactive on $z_{1}$ and $z_{2}$, picking one input and forwarding it on out.

\section{Implementation and Evaluation}

We designed $\lambda_{\leqslant}^{\pi}$ to leverage subtyping and dependent function types, with a formulation close to (a fragment of) Dotty (a.k.a. the future Scala 3 programming language), and its

be assumed w.l.o.g.: if $\Gamma \vdash t: T$ but $\nexists y$ such that $\Gamma \vdash y: U$, we can pick $y^{\prime} \notin \operatorname{dom}(\Gamma)$, extend $\Gamma$ as $\Gamma^{\prime}=\Gamma, y^{\prime}: U$, and get $\Gamma^{\prime} \vdash y^{\prime}: U$ and $\Gamma^{\prime} \vdash t: T$. 
(1) Non-usage of $x_{1}, \ldots, x_{n}$ : none of $x_{1}, \ldots, x_{n}$ is used for output while $t$ runs. (Simple variation: never use $x_{1}, \ldots, x_{n}$ for input)

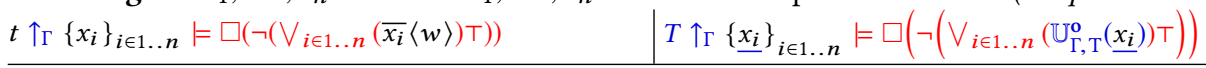

(2) Deadlock-freedom modulo $x_{1}, \ldots, x_{n}: t$ might only use channels $x_{1}, \ldots, x_{n}$ to interact with other processes, and never gets stuck. $t \uparrow_{\Gamma}\left\{x_{i}\right\}_{i \in 1 . . n}\left|=\square\left((\tau) \top \vee \vee_{i \in 1 . . n}\left(x_{i}(w) \cup \overline{x_{i}}\langle w\rangle\right) \top\right)\right| T \uparrow_{\Gamma}\left\{\underline{x}_{i}\right\}_{i \in 1 . . n} \mid=\square\left(-\mathbb{A}_{\tau}\right) \top \wedge \square\left((\tau) \top \vee \vee_{i \in 1 . . n}\left(\left\{\underline{x_{i}}\left(U^{\prime}\right), \underline{x}_{i}\left\langle U^{\prime}\right\rangle \mid\right.\right.\right.$ any $\left.\left.\left.U^{\prime}\right\}\right) \top\right)$

(3) Eventual usage of $x_{1}, \ldots, x_{n}$ : some $x_{i}(i \in 1 . . n)$ is used for output, while $t$ runs. (Simple variations: use some $x_{i}$ for input or communication) $t \uparrow_{\Gamma}\left\{x_{i}\right\}_{i \in 1 . . n}|=\rangle\left(\bigvee_{i \in 1 . . n}\left(\overline{x_{i}}\langle w\rangle\right) \top\right) \quad\left|T \uparrow_{\Gamma}\left\{\underline{x_{i}}\right\}_{i \in 1 . . n}\right|=\left(-\mathbb{A}_{\tau}\right) \top \cup\left(\bigvee_{i \in 1 . . n}\left(\left\{\underline{x_{i}}\left\langle U^{\prime}\right\rangle \mid\right.\right.\right.$ any $\left.\left.\left.U^{\prime}\right\}\right) \top\right)$

(4) Forwarding from $x$ to $y$ : whenever some $z$ is received from $x$, it is eventually forwarded via $y$, before $x$ is used for input again. $t \uparrow_{\Gamma}\{x, y\}|=\square((x(z)) \top \Rightarrow((-x(w)) \top \cup(\bar{y}\langle z\rangle) \top)) \quad| T \uparrow_{\Gamma}\{\underline{x}, \underline{y}\} \mid=\square\left(\left(\left\{S(\underline{z}) \mid S(\underline{z}) \in \mathbb{U}_{\Gamma, \mathrm{T}}^{\mathrm{i}}(\underline{x})\right\}\right) \top \Rightarrow\left(\left(-\left(\mathbb{A}_{\tau} \cup \mathbb{U}_{\Gamma, \mathrm{T}}^{\mathrm{i}}(\underline{x})\right)\right) \top \cup(\underline{\bar{y}}\langle\underline{z}\rangle) \top\right)\right)$

(5) Reactiveness on $x$ : $t$ runs forever, and is always eventually able to receive inputs from $x$ (possibly after a finite number of $\tau$-steps). $t \uparrow_{\Gamma}\{x\} \models \square((\tau) \top \cup(x(w)) \top) \quad\left|T \uparrow_{\Gamma}\left\{\underline{x_{i}}\right\}\right|=\square\left(-\mathbb{A}_{\tau}\right) \top \wedge \square\left((\tau) \top \vee\left(\left\{\underline{x}\left(U^{\prime}\right) \mid\right.\right.\right.$ any $\left.\left.\left.U^{\prime}\right\}\right) \top\right)$

(6) Responsiveness on $x$ : whenever some $z$ is received from $x$, it is eventually used to send a response, before $x$ is used for input again.

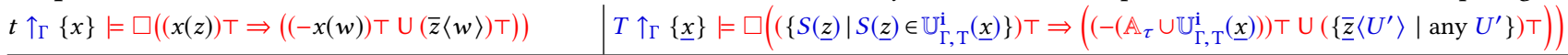

Figure 7. Process verification (Thm. 4.10): the judgement on the left is implied by the companion judgement on the right. Here, $w$ ranges over $\mathbb{V} \cup \mathbb{X}$, and we write $\bar{x}\langle w\rangle$ as shorthand for the (infinite) set of labels $\{\bar{x}\langle w\rangle \mid w \in \mathbb{V} \cup \mathbb{X}\}$ (and similarly for $x(w))$. For brevity, in (4) and (6) we write $(\alpha) \top \Rightarrow \phi$ instead of $(\alpha) \top \Rightarrow(\alpha) \phi$ (i.e., if we observe $\alpha$, then $\phi$ holds afterwards).

foundation $D_{<:}$[2]. This naturally leads to a three-step implementation strategy: (1) internal embedding of $\lambda_{\leqslant}^{\pi}$; (2) actor-based APIs, via syntactic sugar; and (3) compiler plugin for type-level model checking. The result is a software toolkit called Effpi, available at: https://alcestes.github.io/effpi

\subsection{Implementation}

A payoff of the $\lambda_{\leqslant}^{\pi}$ design is that we can implement it as an internal embedded domain-specific language (EDSL) in Dotty: i.e., we can reuse Dotty's syntax and type system, to define: (1) typed communication channels, (2) dedicated methods to render the $\lambda_{\leqslant}^{\pi}$ concurrency primitives (send, recv, $\|$, end), and (3) dedicated classes to render their types (o[...], i $[\ldots]$, $\mathrm{p}[\ldots]$, nil), including the well-formedness and subtyping constraints illustrated in Fig. 4. As usual for internal language embeddings, the Effpi DSL does not directly cause sideeffects: e.g., calling receive(c) $\{x=>P\}$ does not cause an input from channel c. Instead, the receive method returns an object of type $\operatorname{In}[\ldots]$ (corresponding to $\mathrm{i}[\ldots]$ in Def. 3.1), which describes the act of using $c$ to receive a value $v$, and continue as $P\{v / x\}$. Such objects are executed by the Effpi interpreter, according to the $\lambda_{\leqslant}^{\pi}$ semantics (Def. 2.4).

Effpi programs look like def ponger(self: T): T1 $=\{$ the code on the right (which is receive(self) \{ replyTo $\Rightarrow$ ponger from Ex. 2.2): they fol- send(replyTo, "Hi!") >> low the $\lambda \leqslant$ syntax. Also, types end \} \}

are rendered isomorphically: the type " $\underline{x}$ ” in $\lambda \underset{\leqslant}{\pi}$ is rendered as "x. type" in Dotty, and dependent function types become:

$\Pi(\underline{x}: T) \mathbf{o}\left[\underline{y}, \underline{x}, T^{\prime}\right] \rightsquigarrow(\mathrm{x}: \mathrm{T})=>$ Out $\left[y\right.$. type, $\mathrm{x}$. type, $\left.\mathrm{T}^{\prime}\right]$

Thus, the Scala compiler can check the program syntax (§2) and perform type checking (\$3), ensuring type safety (Thm. 3.6). Dotty also supports (local) type inference.

For better usability, Effpi also provides some extensions over $\lambda \frac{\pi}{\leqslant}$, like buffered channels, and a sequencing operator
">>" (see above, and in Fig. 1). Moreover, Effpi simplifies the definition and composition of types-as-protocols by leveraging Dotty's type aliases. E.g., the type of two parallel processes sending an Integer on a same channel can be defined as $U$ (right): no- type $T[X<: \operatorname{Chan}[$ Int $]]=$ Out $[X$, Int $]$ tice how $T$ is reused, type $U[Y<: \operatorname{Chan}[$ Int $]]=\operatorname{Par}[T[Y], T[Y]]$ passing U's parameter. $\operatorname{def} f(x: O C h a n[I n t]): U[x . t y p e]=\ldots$ Also notice how the type of f's argument (x.type) is passed to $U$, and then to $T$ : consequently, the type of $f$ expands into $\operatorname{Par}[$ Out [x.type, Int], Out[x.type, Int $]$.

To guide Effpi's design, we implemented the full "payment with audit" use case from the experimental "session" extension for Akka Typed [41] (cf. §1, code snippet in Fig. 1).

An efficient Effpi interpreter For performance and scalability reasons, many distributed programming toolkits (such as Go, Erlang, and Akka) schedule a (potentially very high) number of logical processes on a limited number of executor threads (e.g., one per CPU core). We follow a similar approach for the Effpi interpreter, leveraging the fact that, in Effpi programs as in $\lambda_{\leqslant}^{\pi}$, input/output actions and their continuations are represented by $\lambda$-terms (closures), that can be easily stored away (e.g., when waiting for an input from a channel), and executed later (e.g., when the desired input becomes available). Thus, we implemented a non-preemptive scheduling system partly inspired by Akka dispatchers [47], with a notable difference: in Effpi, processes yield control (and can be suspended) both when waiting for inputs (as in Akka), and also when sending outputs; this feature requires some sophistication in the scheduling system.

Actor-based API On top of the $\lambda_{\leqslant}^{\pi}$ EDSL, Effpi provides a simplified actor-based API [25], in a flavour similar to Akka Typed [49, 50] (i.e., actors have typed mailboxes and ActorReferences): see Fig. 1. This API models an actor $A$ with mailbox of type $T$, with the intuition in Remark 2.3: 
- $A$ is a process with a unique, implicit input channel $m$, of type $\mathrm{c}^{\mathrm{i}}[T]$ (Def. 3.1). Hence, $A$ can only use $m$ to receive messages of type $T$ - i.e., $m$ is $A$ 's mailbox;

- A receives $T$-typed messages by calling read - which is syntactic sugar for $\operatorname{recv}(m, \ldots)$ (see Fig. 1, and notice that the input channel $m$ is left implicit);

- other processes/actors can send messages to $A$ through its ActorReference $r$ - which is just the output endpoint of its channel/mailbox $m$. The type of $r$ is $\mathrm{c}^{\mathrm{O}}[T]$ (Def. 3.1): it only allows to send messages of type $T$.

To this purpose, Effpi uses Dotty's implicit function types [57]: i.e., type Actor [...] in Fig. 1 hides an input channel.

Type-level model checking The implementation details discussed thus far cover the $\lambda_{\leqslant}^{\pi}$ syntax, semantics, and typing - i.e., §2 and §3. The type-level analysis presented in §4 goes beyond the capabilities of the Dotty compiler; hence, we implement it as a Dotty compiler plugin (i.e., a compiler phase [59]) accessing the typed program AST. The plugin looks for methods annotated with "@effpi . verifier . verify":

$$
\begin{aligned}
& \text { Qeffpi.verifier.verify }(\phi) \\
& \text { def } f(x: \ldots, y: \ldots): T=\ldots
\end{aligned}
$$

Such annotations ask to check if a program of type T satisfies $\phi$, which is a conjunction/disjunctions of the properties from Fig. 7 (left). Note that $T$ can refer to the parameters $x, y, \ldots$ of $f$, and it can be either written by programmers, or inferred by Dotty. Then, the plugin:

1. tries to convert T into a $\lambda_{\leqslant}^{\pi}$ type $T$, as per Def. 3.1;

2. checks if $T=\phi^{\prime}$ holds - where $\phi^{\prime}$ is the companion formula of $\phi$ in Fig. 7 (right). This step uses the mCRL2 model checker [23]: we encode $T$ into an mCRL2 process, ${ }^{2}$ and check if $\phi^{\prime}$ holds;

3. returns an error (located at the code annotation) if steps 1 or 2 fail. Otherwise, the compilation proceeds.

When compilation succeeds, any program of return type $T$ (including $f$ above) enjoys the property $\phi$ at run-time, by Thm. 4.10. This works both when $f$ is implemented, and when it is an unimplemented stub (i.e., when $f$ is defined as "???" in Dotty). This allows to compose the types/protocols of multiple services, and verify their interactions, even without their full implementation. E.g., consider Ex. 2.2, 3.3, and 4.11: a programmer implementing ponger (code above) in Effpi can (a) annotate the method ponger to verify that it is responsive (Fig. 7(6)), and/or (b) annotate an unimplemented stub def $f^{\prime}(\ldots): T^{\prime}=$ ??? with type $T^{\prime}$ matching $T_{p p}$ (Ex. 3.3), to verify that if ponger interacts with any implementation of type $T_{\text {ping }}$, then ponger's self channel is used for output (Fig. 7(3)). Also, a programmer can annotate payment (Fig. 1) to verify that it is reactive and responsive on

\footnotetext{
${ }^{2}$ To obtain an mCRL2 encoding of $T$ with semantics adhering to Def. 4.2, we
} use the encoding into CCS (without restriction) mentioned after Lemma 4.7. its (implicit) mailbox, and Accepts payments after notifying on aud (with a variation of properties (5), (4) in Fig. 7, right).

Known limitations The implementation of our verification approach, outlined above, has three main limitations.

1. It does not check productivity of annotated code: such checks are unsupported in Dotty, and in most programming languages. Hence, programmers must ensure that all functions invoked from their Effpi code eventually return a value - otherwise, liveness properties might not hold at run-time (cf. condition (c1) in §4).

2. It does not verify processes with unbounded parallel components (i.e., with parallel composition under recursion) $;^{3}$ hence, it rejects types having $\mathrm{p}[\ldots, \ldots]$ under $\mu \mathrm{t} . . .$. This does not impact the examples in this paper.

3. It uses iso-recursive types [60, Ch. 21] because, unlike $\lambda_{\leqslant}^{\pi}$ (Def. 3.2), Dotty does not have equi-recursive types.

Limitations 1 and 3 might be avoided by implementing $\lambda \underset{\leqslant}{\pi}$ as a new programming language. However, our Dotty embedding is simpler, and lets Effpi programs access methods and data from any library on the JVM: e.g., Effpi actors/processes can communicate over a network (via Akka Remoting [48]), and with Akka Typed actors.

\subsection{Evaluation}

From §5.1, two factors can hamper Effpi: (1) the run-time impact of its interpreter (speed and memory usage); (2) the verification time of the properties in Fig. 7. We evaluate both.

Run-time benchmarks We adopted a set of benchmarks from the Savina suite [31], with diverse interaction patterns:

- chameneos: $n$ actors ("chameneos") connect to a central broker, who picks pairs and sends them their respective ActorReferences, so they can interact peer-to-peer [34];

- counting: actor $A$ sends $n$ numbers to $B$, who adds them;

- fork-join - creation (FF-C): creation of $n$ new actors, who signal their readiness to interact;

- fork-join - throughput (FF-T): creation of $n$ new actors, and transmission of a sequence of messages to each.

- ping-pong: $n$ pairs of actors exchange requests-responses;

- ring: $n$ actors, connected in a ring, pass each other a token;

- streaming ring: similar to ring, but passing $m$ tokens consecutively (i.e., at most $m$ actors can be active at once).

For all benchmarks, we performed two measurements:

- performance vs. size: how long it takes for the benchmark to complete, depending on the size (i.e., the number of actors, or the number of messages being sent/received);

- memory vs. size: how many times the JVM garbage collector runs, depending on the size of the benchmark and also the maximum memory used before collection.

\footnotetext{
${ }^{3}$ This is because mCRL2 checks formulas of the branching-time $\mu$-calculus, on finite-state systems. We are not aware of model checkers focused on the linear-time $\mu$-calculus, and supporting infinite-state systems.
} 

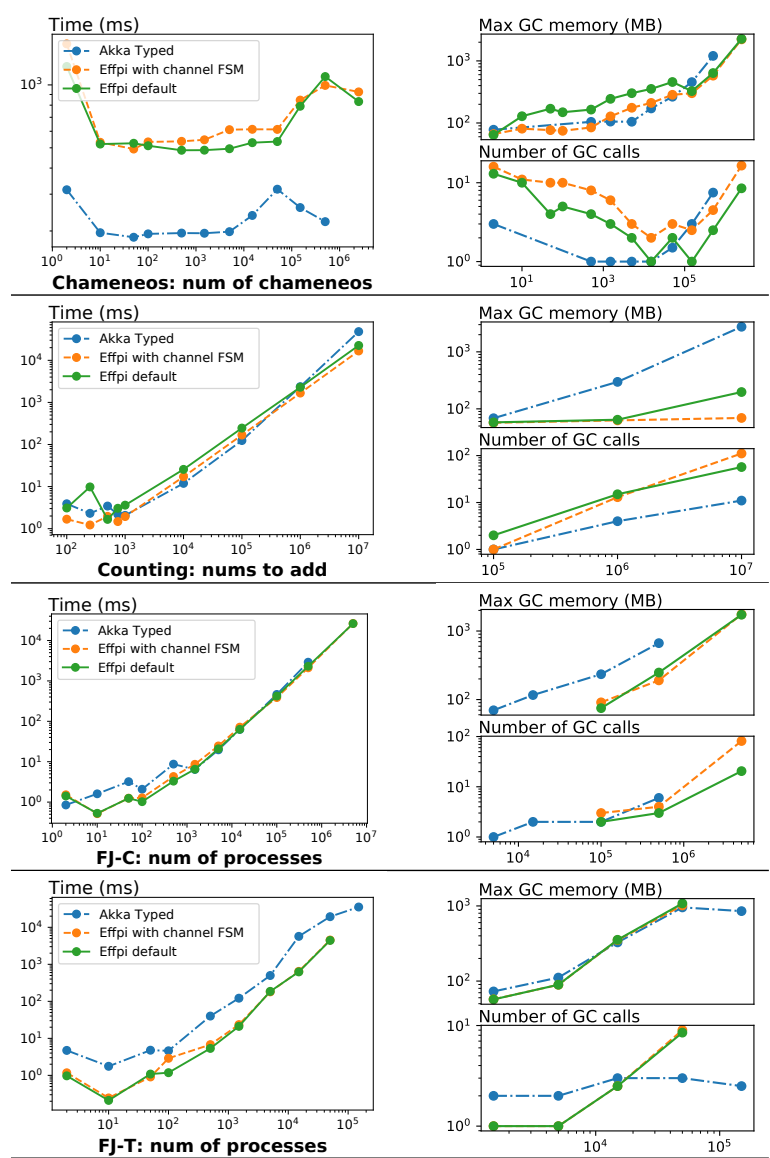

$10^{3} \stackrel{\text { Max GC memory (MB) }}{=}$
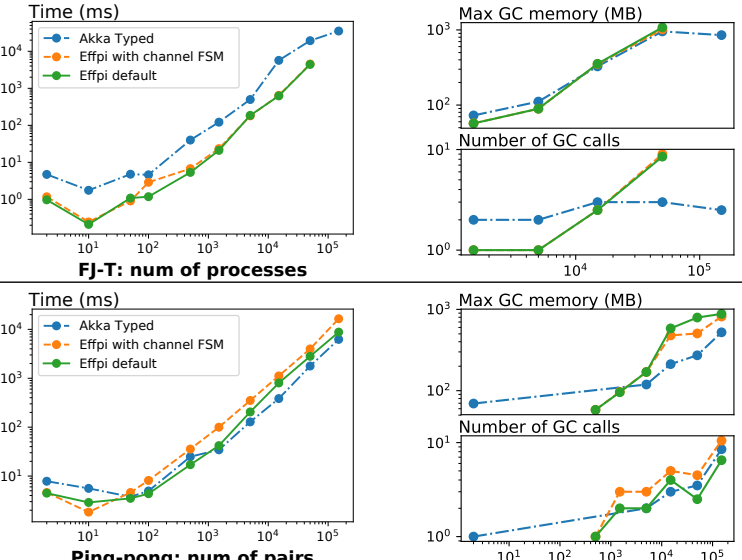

Ping-pong: num of pairs

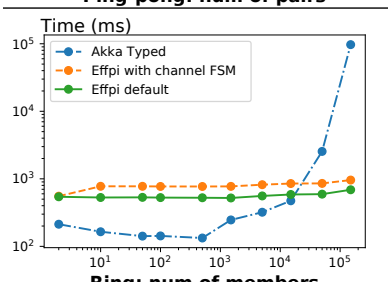

$10^{3}$ Max GC memory (MB)
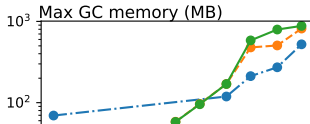

Number of GC calls
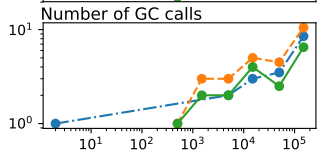

$3 \times 10^{2}$ Max GC memory (MB)
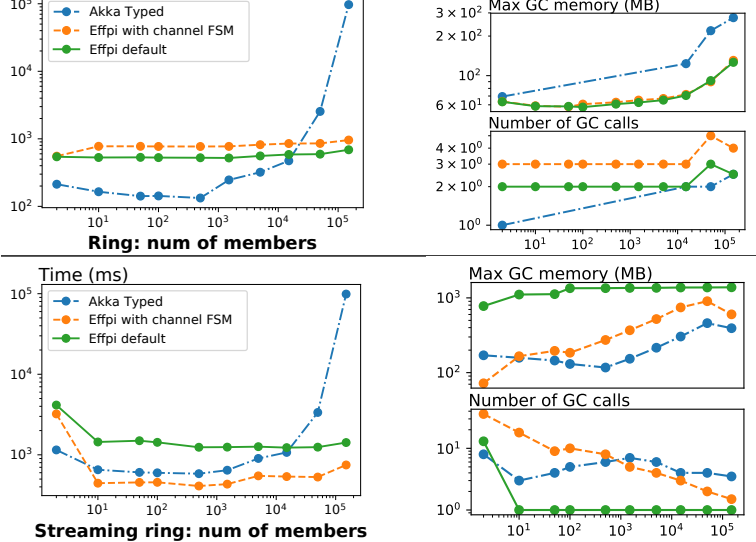

Figure 8. Effpi: mean execution time vs. size (left column, 10 runs, low is better), and memory vs. size (right). Some plots end early (e.g., chameneos+Akka) due to out-of-memory crashes; memory use is plotted when GC runs at least once. (4×Inteli7@3.6GHz, Dotty 0.9.0-RC1, Scala 2.12.7, Akka 2.5.17, 4GB max heap)
The results are in Fig. 8: we compare two instances of the Effpi runtime (with two scheduling policies: "default" and "channel FSM") against Akka, with default setup. Our approach appears viable: Effpi is a research prototype, and still, its performance is not too far from Akka. The negative exception is "chameneos" (Effpi is $\sim 2 \times$ slower); the positive exceptions are fork-join throughput (Effpi is $\sim 2 \times$ faster), and the ring variants (Akka has exponential slowdown).

Model checking benchmarks We evaluated the "extreme cases": the time needed to verify formulas in Fig. 7 on protocols with a large number of states - obtained, e.g., by enlarging the examples in the paper (e.g., composing many parallel ping-pong pairs), aiming at state space explosion. The results are in Fig. 9. Our model checking approach appears viable: it can provide (quasi)real-time verification results, suitable for interactive error reporting on an IDE. Still, model checking performance depends on the size of the model, and on the formula being verified. As expected, our measurements show that verification becomes slower when models are expanded by adding more parallel components, and thus enlarging the state space; they also highlighting that some properties (e.g., our mCRL2 translations of "forwarding" and "responsive") are particularly sensitive to the model size.

\section{Conclusion and Related Work}

We presented a new approach to developing message-passing programs, and verifying their run-time properties. Its cornerstone is a new blend of behavioural+dependent function types, enabling program verification via type-level model checking.

Behavioural types with LTS semantics have been studied in many works [3]: the idea dates back to [56] (for Concurrent ML); type-based verification of temporal logic properties was addressed in $[29,30]$ (for the $\pi$-calculus); recent applications include, e.g., the verification of Go programs [44, 45]. Our key insight is to infuse dependent function types, in order to (1) connect a type variable $\underline{x}$ to a process variable $x$, and (2) gain a form of type-level substitution (Def. 3.1). Item (2), in particular, is not present in previous work; we take advantage of it to compose protocols (Ex. 3.3) and precisely track channel passing and use (Ex. 4.3). Thus, we can verify safety and liveness properties (Fig. 7) while supporting: (1) channel passing, thus covering a core pattern of actorbased programming (Ex. 2.2, Remark 2.3, Ex. 4.11, Fig. 1), and (2) higher-order processes that send/receive mobile code, thus covering an important feature of modern programming toolkits (Ex. 3.4, 4.11). Further, our theory is designed for language embedding: we implemented it in Dotty, and our evaluation supports the viability of the approach $(\S 5)$.

A form of type/channel dependency related to ours is in $[24,78,79]$ : their types depend on process channels, and they check if a process might use a channel $x$ - but cannot say if, when or how $x$ is used, nor verify behavioural properties. 


\begin{tabular}{|c|c|c|c|c|c|c|c|}
\hline & states & deadlock-free & ev-usage & forwarding & non-usage & reactive & responsive \\
\hline Pay $\&$ audit +8 clients & 3328 & true $(0.05 \pm 1.38 \%)$ & true $(0.11 \pm 0.92 \%)$ & false $(6.26 \pm 4.16 \%)$ & false $(0.02 \pm 2.66 \%)$ & true $(1.01 \pm 3.95 \%)$ & true $(15.40 \pm 6.57 \%)$ \\
\hline Pay $\&$ audit +10 clients & 13312 & true $(0.06 \pm 1.65 \%)$ & true $(0.19 \pm 1.07 \%)$ & false $(21.90 \pm 11.19 \%)$ & false $(0.02 \pm 5.55 \%)$ & true $(0.96 \pm 13.22 \%)$ & true $(73.37 \pm 8.28 \%)$ \\
\hline Pay $\&$ audit +12 clients & 53248 & true $(0.07 \pm 1.17 \%)$ & true $(0.23 \pm 1.05 \%)$ & false $(98.72 \pm 12.28 \%)$ & false $(0.02 \pm 2.78 \%)$ & true $(0.99 \pm 2.89 \%)$ & true $(345.22 \pm 8.72 \%)$ \\
\hline Dining philos. (4, deadlock) & 4096 & false $(0.16 \pm 1.41 \%)$ & true $(0.02 \pm 2.02 \%)$ & false $(1.04 \pm 9.84 \%)$ & false $(0.02 \pm 3.55 \%)$ & false $(2.01 \pm 4.79 \%)$ & false $(1.06 \pm 19.65 \%)$ \\
\hline Dining philos. (4, no deadlock) & 4096 & true $(0.16 \pm 0.70 \%)$ & true $(0.02 \pm 2.33 \%)$ & false $(1.19 \pm 28.13 \%)$ & false $(0.02 \pm 1.47 \%)$ & false $(1.91 \pm 14.08 \%)$ & false $(1.07 \pm 19.19 \%)$ \\
\hline Dining philos. (5, deadlock) & 32768 & false $(0.54 \pm 0.80 \%)$ & true $(0.03 \pm 2.46 \%)$ & false $(4.58 \pm 10.54 \%)$ & false $(0.02 \pm 3.55 \%)$ & false $(5.10 \pm 5.78 \%)$ & false $(3.05 \pm 5.11 \%)$ \\
\hline Dining philos. (5, no deadlock) & 32768 & true $(0.55 \pm 1.85 \%)$ & true $(0.03 \pm 1.58 \%)$ & false $(3.05 \pm 4.85 \%)$ & false $(0.02 \pm 3.04 \%)$ & false $(4.21 \pm 8.29 \%)$ & false $(3.01 \pm 1.19 \%)$ \\
\hline Dining philos. (6, deadlock) & 262144 & false $(2.35 \pm 0.51 \%)$ & true $(0.03 \pm 0.87 \%)$ & false $(13.61 \pm 14.39 \%)$ & false $(0.03 \pm 4.22 \%)$ & false $(16.58 \pm 8.22 \%)$ & false $(10.72 \pm 3.88 \%)$ \\
\hline Dining philos. (6, no deadlock) & 262144 & true $(2.37 \pm 0.61 \%)$ & true $(0.03 \pm 2.93 \%)$ & false $(9.20 \pm 5.63 \%)$ & $(0.03 \pm 3.76 \%)$ & false $(17.28 \pm 6.11 \%)$ & false $(6.36 \pm 6.25 \%)$ \\
\hline Ping-pong (6 pairs) & 4096 & true $(0.05 \pm 1.68 \%)$ & true $(0.01 \pm 3.92 \%)$ & false $(0.95 \pm 14.43 \%)$ & $(0.01 \pm 16.42 \%)$ & $(0.98 \pm 6.02 \%)$ & $0.98 \pm 5.34 \%)$ \\
\hline Ping-pong (6 pairs, responsive) & 46656 & true $(0.26 \pm 2.65 \%)$ & & $(1.05 \pm 13.51 \%)$ & & & \\
\hline Ping-pong (8 pairs) & 65536 & true $(0.23 \pm 0.82 \%)$ & true $(0.01 \pm 3.07 \%)$ & false $(2.00 \pm 1.25 \%)$ & false $(0.01 \pm 3.27 \%)$ & $2.01 \pm 2.48 \%)$ & false $(1.53 \pm 30.27 \%)$ \\
\hline Ping-pong (8 pairs, responsive) & 1679616 & true $(1.60 \pm 1.90 \%)$ & true $(0.03 \pm 2.43 \%)$ & false $(6.89 \pm 3.14 \%)$ & false $(0.03 \pm 5.62 \%)$ & $(4.58 \pm 9.96 \%)$ & $(9.39 \pm 6.48 \%)$ \\
\hline Ping-pong (10 pairs) & 1048576 & true $(2.40 \pm 1.63 \%)$ & true $(0.02 \pm 2.35 \%)$ & false $(8.63 \pm 13.49 \%)$ & false $(0.01 \pm 1.69 \%)$ & false $(9.53 \pm 10.27 \%)$ & false $(1.99 \pm 2.69 \%)$ \\
\hline Ping-pong (10 pairs, responsive) & $>2 \times 10^{6}$ & true $(8.74 \pm 10.83 \%)$ & true $(0.04 \pm 2.66 \%)$ & false $(17.00 \pm 1.62 \%)$ & false $(0.03 \pm 1.39 \%)$ & false $(23.49 \pm 4.76 \%)$ & true $(50.97 \pm 5.80 \%)$ \\
\hline Ring (10 elements) & 2048 & true $(0.01 \pm 3.58 \%)$ & true $(0.01 \pm 3.82 \%)$ & true $(11.34 \pm 1.48 \%)$ & false $(0.01 \pm 2.44 \%)$ & true $(7.81 \pm 0.35 \%)$ & false $(1.00 \pm 1.10 \%)$ \\
\hline Ring (15 elements) & 65536 & true $(0.02 \pm 1.57 \%)$ & true $(0.02 \pm 1.56 \%)$ & true $(562.48 \pm 4.72 \%)$ & false $(0.01 \pm 1.79 \%)$ & true $(407.47 \pm 7.13 \%)$ & false $(108.61 \pm 3.10 \%)$ \\
\hline Ring (10 elements, 3 tokens) & 4096 & true $(0.06 \pm 3.14 \%)$ & true $(0.01 \pm 1.72 \%)$ & true $(23.79 \pm 9.10 \%)$ & false $(0.01 \pm 4.07 \%)$ & true $(15.53 \pm 0.38 \%)$ & false $(1.99 \pm 8.18 \%)$ \\
\hline Ring (15 elements, 3 tokens) & 131072 & true $(0.39 \pm 0.60 \%)$ & true $(0.01 \pm 1.44 \%)$ & true $(1146.57 \pm 2.11 \%)$ & false $(0.01 \pm 2.19 \%)$ & true $(827.58 \pm 1.00 \%)$ & false $(2.01 \pm 7.92 \%)$ \\
\hline
\end{tabular}

Figure 9. Behavioural property verification: outcome (true/false) and average time (seconds \pm std. dev.). The number of states

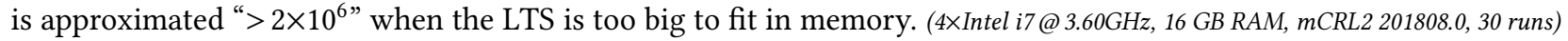

Various $\pi$-calculus type systems specialise on accurate (dead)lock-freedom analysis, e.g., [36-39, 58]. [13] type-checks actors with unordered mailboxes, carrying messages of different types; it ensures deadlock-freedom, and (assuming termination) message consumption. Unlike ours, the works above do not support an extensible set of $\mu$-calculus properties (Fig. 7), nor address higher-order processes. Although our actors are similar to Akka Typed (with single-type mailboxes), we conjecture that our types also support actors like [13], with decidable verification (by Lemma. 4.7).

Our protocols-as-types are related to session types [11, 26, 27, 69], and their combination with value-dependent and indexed types [10, 14, 75-77]; session types have inspired various implementations [3], also in Scala [65-68]. Our theory has a different design, yielding different features. On the one hand, we do not have an explicit external choice construct (we plan to integrate it via match types [17], but leave it as future work); on the other hand, we can verify liveness properties across interleaved use of multiple channels (more liberally than session types [12]), and we are not limited to linear/confluent protocols: e.g., $T=$ $\mathrm{p}\left[\mathrm{p}\left[\mathrm{o}[\underline{x}, \underline{y}, T], \mathrm{o}\left[\underline{x}, \underline{z}, T^{\prime}\right]\right], \mathrm{i}\left[\underline{x}, \Pi\left(\underline{z^{\prime}}: \mathrm{c}^{\mathrm{io}}[\mathrm{int}]\right) U\right]\right]$ types parallel processes with a race on channel $\underline{x}$; we can verify such processes, capturing that either $y$ or $\underline{z}$ may replace $\underline{z^{\prime}}$ in the $U$-typed continuation. This covers locking/mutex protocols, allowing, e.g., to implement and verify Dijkstra's dining philosopher problem (mentioned in Fig. 9). [4] extends linear logic-based session types with shared channels: it adds non-determinism, weakening deadlock-freedom guarantees.

Outside the realm of process calculi, various works tackle the problem of protocol-aware verification, e.g., [40, 71, 74]. We share similar goals, although we adopt a different theory and design, leading to different tradeoffs: crucially, the works above develop new languages, or build upon a powerful dependently-typed host language (Coq) with interactive proofs, to support rich representations of protocol state. We, instead, aim at Dotty embedding (with limited type dependencies) and automated verification of process properties (via type-level model checking); hence, our protocols and logic are action-based, to ensure decidability (Lemma 4.7). Our approach covers many stateful protocols (e.g., locking/mutex, mentioned above); but beyond this, a finer type-level representation of state may make model checking undecidable [19], thus requiring decidability conditions, or novel heuristic/interactive proof techniques. This topic can foster exciting future work, and a cross-pollination of results between the realms of protocol-aware verification, and process calculi.

Future work We will study $\lambda_{\leqslant}^{\pi}$ embeddings in other programming languages - although only Dotty provides both subtyping and dependent function types. We will extend the supported properties in Fig. 7, and study how to improve their verification, along three directions: 1 . increase speed, trying more mCRL2 options, and tools like LTSmin [35]; 2. support infinite-state systems, trying tools like BFC [33] (that does not cover the linear-time $\mu$-calculus in Def. 4.6, but is used e.g. in [15] to verify safety properties of actor programs); 3. introduce assume-guarantee reasoning for typelevel model checking, inspired by [62]. The Effpi runtime system can be optimised: we will attempt its integration with Akka Dispatchers [47], and explore other (non-preemptive) scheduling strategies, e.g., work stealing [1,5].

Acknowledgements Thanks to the anonymous reviewers for their remarks, to Sung-Shik Jongmans for his comments, and to Raymond $\mathrm{Hu}$ for testing the artifact. Work partially supported by EPSRC projects EP/K034413/1, EP/K011715/1, EP/L00058X/1, EP/N027833/1, EP/N028201/1. 


\section{References}

[1] Umut A. Acar, Arthur Chargueraud, and Mike Rainey. 2013. Scheduling Parallel Programs by Work Stealing with Private Deques. In PPoPP. https://doi.org/10.1145/2442516.2442538

[2] Nada Amin, Samuel Grütter, Martin Odersky, Tiark Rompf, and Sandro Stucki. 2016. The Essence of Dependent Object Types. In A List of Successes That Can Change the World - Essays Dedicated to Philip Wadler on the Occasion of His 60th Birthday. https://doi.org/10.1007/ 978-3-319-30936-1_14

[3] Davide Ancona, Viviana Bono, Mario Bravetti, Joana Campos, Giuseppe Castagna, Pierre-Malo Deniélou, Simon J. Gay, Nils Gesbert, Elena Giachino, Raymond Hu, Einar Broch Johnsen, Francisco Martins, Viviana Mascardi, Fabrizio Montesi, Rumyana Neykova, Nicholas $\mathrm{Ng}$, Luca Padovani, Vasco T. Vasconcelos, and Nobuko Yoshida. 2017. Behavioral Types in Programming Languages. Foundations and Trends in Programming Languages 3(2-3) (2017). https://doi.org/10.1561/ 2500000031

[4] Stephanie Balzer and Frank Pfenning. 2017. Manifest Sharing with Session Types. Proc. ACM Program. Lang. 1, ICFP, Article 37 (2017). https://doi.org/10.1145/3110281

[5] Robert D. Blumofe and Charles E. Leiserson. 1999. Scheduling Multithreaded Computations by Work Stealing. F. ACM 46, 5 (1999), 29. https://doi.org/10.1145/324133.324234

[6] Julian Bradfield and Colin Stirling. 2007. Modal $\mu$-calculi. In Handbook of Modal Logic. Elsevier. https://doi.org/10.1016/S1570-2464(07) 80015-2

[7] Nadia Busi, Maurizio Gabbrielli, and Gianluigi Zavattaro. 2009. On the expressive power of recursion, replication and iteration in process calculi. Mathematical Structures in Computer Science 19, 6 (2009), 11911222. https://doi.org/10.1017/S096012950999017X

[8] L. Cardelli, S. Martini, J.C. Mitchell, and A. Scedrov. 1994. An Extension of System F with Subtyping. Information and Computation 109, 1 (1994). https://doi.org/10.1006/inco.1994.1013

[9] Luca Cardelli and Peter Wegner. 1985. On Understanding Types, Data Abstraction, and Polymorphism. Comput. Surveys 17, 4 (1985), 53. https://doi.org/10.1145/6041.6042

[10] David Castro, Raymond Hu, Sung-Shik Jongmans, Nicholas Ng, and Nobuko Yoshida. 2019. Distributed Programming Using Roleparametric Session Types in Go: Statically-typed Endpoint APIs for Dynamically-instantiated Communication Structures. Proc. ACM Program. Lang. 3, POPL, Article 29 (2019). https://doi.org/10.1145/3290342

[11] Mario Coppo, Mariangiola Dezani-Ciancaglini, Luca Padovani, and Nobuko Yoshida. 2015. A Gentle Introduction to Multiparty Asynchronous Session Types. In Formal Methods for Multicore Programming. https://doi.org/10.1007/978-3-319-18941-3_4

[12] Mario Coppo, Mariangiola Dezani-Ciancaglini, Nobuko Yoshida, and Luca Padovani. 2015. Global Progress for Dynamically Interleaved Multiparty Sessions. MSCS 760 (2015). https://doi.org/10.1017/ S0960129514000188

[13] Ugo de'Liguoro and Luca Padovani. 2018. Mailbox Types for Unordered Interactions. In ECOOP. https://doi.org/10.4230/LIPIcs.ECOOP.2018.15

[14] Pierre-Malo Deniélou, Nobuko Yoshida, Andi Bejleri, and Raymond Hu. 2012. Parameterised Multiparty Session Types. Logical Methods in Computer Science 8, 4 (2012). https://doi.org/10.2168/LMCS-8(4:6)2012

[15] Emanuele D’Osualdo, Jonathan Kochems, and C. H. Luke Ong. 2013. Automatic Verification of Erlang-Style Concurrency. In Static Analysis. Berlin, Heidelberg. https://doi.org/10.1007/978-3-642-38856-9_24

[16] Dotty developers. 2019. Dotty documentation: dependent function types. https://dotty.epfl.ch/docs/reference/new-types/ dependent-function-types.html.

[17] Dotty developers. 2019. Dotty documentation: match types. http: //dotty.epfl.ch/docs/reference/new-types/match-types.html.

[18] Ericsson. 2019. The Erlang/OTP Programming Language and Toolkit. http://erlang.org/.
[19] Javier Esparza. 1994. On the decidability of model checking for several $\mu$-calculi and Petri nets. In Trees in Algebra and Programming - CAAP. https://doi.org/10.1007/BFb0017477

[20] Javier Esparza. 1997. Decidability of model checking for infinite-state concurrent systems. Acta Informatica 34, 2 (1997). https://doi.org/10. $1007 / \mathrm{s} 002360050074$

[21] Jürgen Giesl, Matthias Raffelsieper, Peter Schneider-Kamp, Stephan Swiderski, and René Thiemann. 2011. Automated Termination Proofs for Haskell by Term Rewriting. TOPLAS 33, 2, Article 7 (2011). https: //doi.org/10.1145/1890028.1890030

[22] Ursula Goltz. 1990. CCS and Petri nets. In Semantics of Systems of Concurrent Processes. https://doi.org/10.1007/3-540-53479-2_14

[23] Jan Friso Groote and Mohammad Reza Mousavi. 2014. Modeling and Analysis of Communicating Systems. The MIT Press.

[24] Matthew Hennessy, Julian Rathke, and Nobuko Yoshida. 2005. safeDpi: a language for controlling mobile code. Acta Informatica 42, 4-5 (2005). https://doi.org/10.1007/s00236-005-0178-y

[25] Carl Hewitt, Peter Bishop, Irene Greif, Brian Smith, Todd Matson, and Richard Steiger. 1973. Actor Induction and Meta-evaluation. In POPL. https://doi.org/10.1145/512927.512942

[26] Kohei Honda. 1993. Types for Dyadic Interaction. In CONCUR. https: //doi.org/10.1007/3-540-57208-2_35

[27] Kohei Honda, Nobuko Yoshida, and Marco Carbone. 2008. Multiparty asynchronous session types. In POPL. https://doi.org/10.1145/1328438. 1328472 Journal version in [28].

[28] Kohei Honda, Nobuko Yoshida, and Marco Carbone. 2016. Multiparty Asynchronous Session Types. f. ACM 63, 1, Article 9 (2016). https: //doi.org/10.1145/2827695

[29] Atsushi Igarashi and Naoki Kobayashi. 2001. A Generic Type System for the Pi-calculus. In POPL. https://doi.org/10.1145/360204.360215

[30] Atsushi Igarashi and Naoki Kobayashi. 2004. A generic type system for the $\pi$-calculus. TCS 311, 1 (2004). https://doi.org/10.1016/ S0304-3975(03)00325-6

[31] Shams M. Imam and Vivek Sarkar. 2014. Savina - An Actor Benchmark Suite: Enabling Empirical Evaluation of Actor Libraries (AGERE!). https://doi.org/10.1145/2687357.2687368

[32] Alan Jeffrey. 2001. A Symbolic Labelled Transition System for Coinductive Subtyping of $F_{\mu}<$ Types. In LICS. https://doi.org/10.1109/LICS. 2001.932508

[33] Alexander Kaiser, Daniel Kroening, and Thomas Wahl. 2015. Bfc - A Widening Approach to Multi-Threaded Program Verification. http: //www.cprover.org/bfc/.

[34] Claude Kaiser and Jean-Francois Pradat-Peyre. 2003. Chameneos, a concurrency game for Java, Ada and others. In ACS/IEEE Int. Conf. on Computer Systems and Applications. Book of abstracts. https://doi.org/ 10.1109/AICCSA.2003.1227495

[35] Gijs Kant, Alfons Laarman, Jeroen Meijer, Jaco van de Pol, Stefan Blom, and Tom van Dijk. 2015. LTSmin: High-Performance LanguageIndependent Model Checking. In TACAS. https://doi.org/10.1007/ 978-3-662-46681-0_61

[36] Naoki Kobayashi. 1998. A Partially Deadlock-Free Typed Process Calculus. TOPLAS 20, 2 (1998). https://doi.org/10.1145/276393.278524

[37] Naoki Kobayashi. 2006. A New Type System for Deadlock-Free Processes. In CONCUR. https://doi.org/10.1007/11817949_16

[38] Naoki Kobayashi and Cosimo Laneve. 2017. Deadlock analysis of unbounded process networks. Information and Computation 252 (2017). https://doi.org/10.1016/j.ic.2016.03.004

[39] Naoki Kobayashi and Davide Sangiorgi. 2010. A hybrid type system for lock-freedom of mobile processes. TOPLAS 32, 5 (2010). https: //doi.org/10.1145/1745312.1745313

[40] Morten Krogh-Jespersen, Amin Timany, and Lars Birkedal Marit Edna Ohlenbusch. 2018. Aneris: A Logic for Node-Local, Modular Reasoning of Distributed Systems. https://iris-project.org/pdfs/ 2019-aneris-submission.pdf. Unpublished draft. 
[41] Roland Kuhn. 2017. Akka Typed Session. https://github.com/rkuhn/ akka-typed-session.

[42] Roland Kuhn. 2017. Akka Typed Session: audit example. https://github. com/rkuhn/akka-typed-session/blob/master/src/test/scala/com/ rolandkuhn/akka_typed_session/auditdemo/ProcessBased.scala.

[43] Leslie Lamport. 1977. Proving the Correctness of Multiprocess Programs. IEEE Transactions on Software Engineering SE-3, 2 (March 1977). https://doi.org/10.1109/TSE.1977.229904

[44] Julien Lange, Nicholas Ng, Bernardo Toninho, and Nobuko Yoshida. 2017. Fencing off go: liveness and safety for channel-based programming. In POPL. https://doi.org/10.1145/3093333.3009847

[45] Julien Lange, Nicholas Ng, Bernardo Toninho, and Nobuko Yoshida. 2018. A static verification framework for message passing in Go using behavioural types. In ICSE. https://doi.org/10.1145/3180155.3180157

[46] Lightbend, Inc. 2017. Akka Typed: Protocols. https://akka.io/blog/ 2017/05/12/typed-protocols.

[47] Lightbend, Inc. 2019. Akka Dispatchers documentation. https: //doc.akka.io/docs/akka/2.5/dispatchers.html.

[48] Lightbend, Inc. 2019. Akka remoting documentation. https://doc.akka. io/docs/akka/2.5/remoting.html.

[49] Lightbend, Inc. 2019. The Akka toolkit and runtime. http://akka.io/.

[50] Lightbend, Inc. 2019. Akka Typed documentation. https://doc.akka. io/docs/akka/2.5/typed/index.html.

[51] Barbara H. Liskov and Jeannette M. Wing. 1994. A Behavioral Notion of Subtyping. TOPLAS 16, 6 (1994). https://doi.org/10.1145/197320.197383

[52] Heather Miller, Philipp Haller, and Martin Odersky. 2014. Spores: A Type-Based Foundation for Closures in the Age of Concurrency and Distribution. In ECOOP. https://doi.org/10.1007/978-3-662-44202-9_13

[53] Robin Milner. 1989. Communication and Concurrency. Prentice-Hall, Inc.

[54] Robin Milner. 1999. Communicating and Mobile Systems: the $\pi$-Calculus. Cambridge University Press.

[55] Robin Milner, Joachim Parrow, and David Walker. 1992. A Calculus of Mobile Processes, Parts I and II. Information and Computation 100, 1 (1992). https://doi.org/10.1016/0890-5401(92)90008-4

[56] Hanne Riis Nielson and Flemming Nielson. 1994. Higher-order Concurrent Programs with Finite Communication Topology (Extended Abstract). In POPL. https://doi.org/10.1145/174675.174538

[57] Martin Odersky, Olivier Blanvillain, Fengyun Liu, Aggelos Biboudis, Heather Miller, and Sandro Stucki. 2017. Simplicitly: Foundations and Applications of Implicit Function Types. Proc. ACM Program. Lang. 2, POPL, Article 42 (2017). https://doi.org/10.1145/3158130

[58] Luca Padovani. 2014. Deadlock and lock freedom in the linear $\pi$ calculus. In CSL-LICS. https://doi.org/10.1145/2603088.2603116

[59] Dmitry Petrashko, Ondřej Lhoták, and Martin Odersky. 2017. Miniphases: Compilation Using Modular and Efficient Tree Transformations. In PLDI. https://doi.org/10.1145/3062341.3062346

[60] Benjamin C. Pierce. 2002. Types and programming languages. MIT Press.

[61] Benjamin C. Pierce and Davide Sangiorgi. 1996. Typing and Subtyping for Mobile Processes. Mathematical Structures in Computer Science 6, 5 (1996).

[62] Sriram K. Rajamani and Jakob Rehof. 2001. A Behavioral Module System for the Pi-Calculus. In SAS. https://doi.org/10.1007/3-540-47764-0_
22

[63] Davide Sangiorgi and David Walker. 2001. The $\pi$-calculus: a Theory of Mobile Processes. Cambridge University Press.

[64] Alceste Scalas, Elias Benussi, and Nobuko Yoshida. 2019. Effpi website. https://alcestes.github.io/effpi.

[65] Alceste Scalas, Ornela Dardha, Raymond Hu, and Nobuko Yoshida. 2017. A Linear Decomposition of Multiparty Sessions for Safe Distributed Programming. In ECOOP. https://doi.org/10.4230/LIPIcs.ECOOP. 2017.24

[66] Alceste Scalas, Ornela Dardha, Raymond Hu, and Nobuko Yoshida. 2017. A Linear Decomposition of Multiparty Sessions for Safe Distributed Programming (Artifact). Dagstuhl Artifacts Series 3, 1 (2017). https://doi.org/10.4230/DARTS.3.2.3

[67] Alceste Scalas and Nobuko Yoshida. 2016. Lightweight Session Programming in Scala. In ECOOP. https://doi.org/10.4230/LIPIcs.ECOOP. 2016.21

[68] Alceste Scalas and Nobuko Yoshida. 2016. Lightweight Session Programming in Scala (Artifact). Dagstuhl Artifacts Series 2, 1 (2016). https://doi.org/10.4230/DARTS.2.1.11

[69] Alceste Scalas and Nobuko Yoshida. 2019. Less is More: Multiparty Session Types Revisited. Proc. ACM Program. Lang. 3, POPL, Article 30 (Jan. 2019). https://doi.org/10.1145/3290343

[70] Alceste Scalas, Nobuko Yoshida, and Elias Benussi. 2019. Verifying message-passing programs with dependent behavioural types. https: //www.doc.ic.ac.uk/research/technicalreports/2019/\#1 DoC Technical report 2019/1.

[71] Ilya Sergey, James R. Wilcox, and Zachary Tatlock. 2018. Programming and proving with distributed protocols. PACMPL 2, POPL (2018). https: //doi.org/10.1145/3158116

[72] Antal Spector-Zabusky, Joachim Breitner, Christine Rizkallah, and Stephanie Weirich. 2018. Total Haskell is Reasonable Coq. In CPP. https://doi.org/10.1145/3167092

[73] Colin Stirling. 2001. Modal and Temporal Properties of Processes. Springer-Verlag New York, Inc., New York, NY, USA.

[74] Marcelo Taube, Giuliano Losa, Kenneth L. McMillan, Oded Padon, Mooly Sagiv, Sharon Shoham, James R. Wilcox, and Doug Woos. 2018. Modularity for decidability of deductive verification with applications to distributed systems. In PLDI. https://doi.org/10.1145/3192366. 3192414

[75] Bernardo. Toninho, Luís. Caires, and Frank Pfenning. 2011. Dependent session types via intuitionistic linear type theory. In PPDP. https: //doi.org/10.1145/2003476.2003499

[76] Bernardo Toninho and Nobuko Yoshida. 2017. Certifying data in multiparty session types. fournal of Logical and Algebraic Methods in Programming 90, C (2017). https://doi.org/j.jlamp.2016.11.005

[77] Bernardo Toninho and Nobuko Yoshida. 2018. Depending on Session-Typed Processes. In FoSSaCS. https://doi.org/10.1007/ 978-3-319-89366-2_7

[78] Nobuko Yoshida. 2004. Channel dependent types for higher-order mobile processes. In POPL. https://doi.org/10.1145/964001.964014

[79] Nobuko Yoshida and Matthew Hennessy. 2002. Assigning Types to Processes. Information and Computation 174, 2 (2002). https://doi.org/ 10.1006/inco.2002.3113 